\title{
A probabilistic approach to demixing odors
}

\author{
Agnieszka Grabska-Barwińska ${ }^{1,2}$, Simon Barthelmé ${ }^{3}$, Jeff Beck ${ }^{4}$, \\ Zachary F. Mainen ${ }^{5}$, Alexandre Pouget ${ }^{1,6,7,8}$ and Peter E. Latham ${ }^{1,8}$ \\ ${ }^{1}$ Gatsby Computational Neuroscience Unit, UCL, London, UK \\ ${ }^{2}$ Google DeepMind, 5 New Street Square, London EC4A 3TW, UK \\ ${ }^{3}$ CNRS, Gipsa-lab, Grenoble, France \\ ${ }^{4}$ Department of Neurobiology, Duke University Medical School, Durham, US \\ ${ }^{5}$ Champalimaud Centre for the Unknown, Lisbon, Portugal \\ ${ }^{6}$ University of Geneva, Geneva, Switzerland \\ ${ }^{7}$ University of Rochester, Rochester, US \\ ${ }^{8}$ These authors contributed equally to the work
}

September 19, 2016

\begin{abstract}
The olfactory system faces a hard problem: based on noisy information from olfactory receptor neurons (the neurons that transduce chemicals to neural activity), it has to figure out which odors are present in the world. Odors almost never occur in isolation, and different odors excite overlapping populations of olfactory receptor neurons, so the central challenge of the olfactory system is to demix its input. Because of noise and the large number of possible odors, demixing is a fundamentally probabilistic inference task. We propose that the early olfactory system uses approximate Bayesian inference to solve it. The computations involve a dynamical loop between the olfactory bulb and the piriform cortex, with cortex explaining incoming activity from the olfactory receptor neurons in terms of a mixture of odors. The model is compatible with known anatomy and physiology, including pattern decorrelation, and it performs better than other models at demixing odors.
\end{abstract}

\section{INTRODUCTION}

The olfactory system has evolved to process information about chemicals in the environment. Much is known about the physiological side of this processing, especially in the early stages. ${ }^{1}$ At the very first stage, neurons in the nasal epithelium, called olfactory receptor neurons (ORNs), transduce chemicals in the air into electrical signals. Each ORN expresses exactly one type of olfactory receptor, and in mammals there are about 1000 different types. The question we address here is: how does the brain extract olfactory percepts from the ORN activity? More simply, how does it answer questions like: given the relatively complex mixture of chemicals just inhaled, which odors are present? (We use "odor" to refer to the olfactory percept corresponding to a particular object, as in the "the odor of an orange", as opposed to the multiple chemicals, or odorants, that are released by an orange.) 
The problem of inferring olfactory percepts from the ORN signals is difficult for several reasons. First, it is rarely the case that a single odor dominates the environment. Instead, multiple odors are typically present: in a restaurant there are many different dishes, in a forest many different plants, etc. Thus, the task of the olfactory system is more often segmentation than recognition, at least outside of an experimental laboratory. And even when the task is to recognize a single odor, that typically must be done against a background of other odors. ${ }^{2}$ Second, ORNs respond to a broad range of odors, ${ }^{3}$ so information about the olfactory scene is distributed across many neurons. Finally, because neural responses are stochastic, the same odor never elicits the same pattern of activity twice.

For all these reasons, olfaction is fundamentally a probabilistic inference task. Based on this, we hypothesize that when faced with an olfactory scene, the olfactory system computes a probability distribution over the possible odors. Although it is not known whether olfactory neurons encode probabilities, there is very strong evidence that other sensory modalities do, ${ }^{4}$ so this is a reasonable hypothesis. And there are good reasons to keep track of probabilities. For example, suppose you smell a fruit and conclude that there's an $80 \%$ chance it's a grapefruit and a $20 \%$ chance it's an orange. Suppose you then look at the fruit and, based on the image, conclude that there's a 5\% chance it's a grapefruit and a $95 \%$ chance it's an orange. Such cross-modal disagreement is easily resolved using the rules of probabilistic inference (assuming equal a priori probabilities, there's about a 17\% chance it's a grapefruit). But it is only because the probabilities were known that an optimal decision - about, say, whether to eat the fruit - could be made. If the two modalities had returned binary answers ("it's a grapefruit" and "it's an orange"), there would be no principled way to resolve the conflict.

Here we present a model of how the early olfactory system - the olfactory bulb, along with the piriform cortex - could "demix" odors. More specifically, we show that the early olfactory system can, based on a single sniff, compute a probability distribution over the concentration of each possible odor via a dynamic process involving the olfactory bulb and the piriform cortex. Previous work on demixing has either assumed that only one odor is detected on each sniff, ${ }^{5-7}$ or that different odors have different temporal patterns. ${ }^{8,9}$ Our model is unique in that it both treats odors probabilistically and is capable of demixing multiple, temporally homogeneous, odors in a single sniff.

\section{RESULTS}

\section{A probabilistic model of olfaction}

Essentially all probabilistic models of sensory processing proceed in three steps, and olfaction is no different: 1) specify an encoding model - a probabilistic mapping from odors to neural activity, 2) specify a prior probability over odors, and 3) use Bayes' theorem to invert the model and compute a probability distribution over odors given neural activity. Applying this procedure results in a set of equations that constitutes our inference algorithm - the algorithm for transforming neural activity into a probability distribution. In Online Methods, Sec. 3, we provide a detailed description of the encoding model, the prior, and the method for inverting Bayes' theorem. Here we sketch the main ideas.

The encoding model specifies the activity of each ORN receptor type given the set of 
concentrations in the world. For that we use a relatively simple model: the firing rate of ORN receptor type $i$, denoted $\nu_{i}$, is a weighted sum of the concentrations,

$$
\nu_{i}=\nu_{0, i}+\Delta t^{-1} \sum_{j=1}^{K} w_{i j} c_{j}
$$

where $c_{j}$ is taken to be $\log$ concentration, chosen so that $c_{j}=0$ corresponds to a concentration so low that it is undetectable, $\nu_{0, i}$ is the background firing rate of ORN receptor type $i$, and $\Delta t$ is the time window for counting spikes (as we discuss shortly). We assume that the neurons fire with Poisson statistics, as has been observed, at least approximately; ${ }^{10}$ thus, in our model the input to the olfactory bulb is a set of Poisson spike trains.

The second step of probabilistic modeling is to determine the prior probability distribution over concentration (the distribution over $c_{j}$ ). Here we make two assumptions. The first is that only a small number of odors are present at a time, meaning only a small number of the $c_{j}$ are nonzero. The second is that odors occur independently of each other. While the former is reasonable (we rarely detect more than a handful of odors at any one time) the latter is not correct. That's because odors tend to be correlated; e.g., the set of odors one expects in a restaurant are different from those one expects in a forest. However, modeling those dependencies would require a complex, hierarchical prior. While such a prior is, ultimately, important for a complete understanding of olfaction, it adds complexity without changing the basic story; we thus leave it for future work.

We can satisfy both assumptions by combining, for each odor, a smooth function, which describes concentrations above detection threshold, with a point distribution whose total probability corresponds to the fraction of time concentrations are so low as to be undetectable. This gives us a prior of the form

$$
p(\mathbf{c})=\prod_{j}\left[\left(1-p_{\text {prior }}\right) \delta\left(c_{j}\right)+p_{\text {prior }} \frac{e^{-c_{j} / \beta_{\text {prior }}}}{\beta_{\text {prior }}}\right]
$$

where $p_{\text {prior }}$ is the prior probability that any particular odor is present, $\beta_{\text {prior }}$ sets the characteristic scale of the concentrations, and $\delta\left(c_{j}\right)$ is a delta-function (the point mass). In our simulations we use $\beta_{\text {prior }}=3$ and, $p_{\text {prior }}=3 / K$ where $K(=640)$ is the number of odors; the latter implies that there are, on average, 3 odors present in any particular olfactory scene. The precise shape of the distribution isn't so important. What is important is that the expected number of odors is small, so that the olfactory system infers mixtures with a small number of odors.

The third step is to invert the generative model - combine the prior (Eq. (2)) with the encoding model (Eq. (1)) - to determine the probability that any particular odor is present. To do that, the olfactory system needs to know the possible odors in the world and the set of weights, $w_{i j}$, that transform those odors to ORN activity. In a full treatment, these would be learned; here we assume learning has already occurred. Or, more precisely, we assume that a subset of the possible odors, and their corresponding weights, have been learned. We refer to the learned subset as "known" odors. All other odors are "unknown".

Exact inference in this model is not feasible, as computation time is exponential in the number of odors; this is typical of Bayesian inference problems in realistic settings. 
We therefore use an approximate algorithm, which, as we'll see, can be implemented in neural circuitry consistent with known anatomy and physiology. ${ }^{11,12}$ The algorithm finds the independent distribution that is as close as possible to the true one. As we show in Online Methods, Sec. 3 (in particular Eq. (21a)), this results in a posterior distribution for the concentration of each odor. The posterior for odor $j$ has the form

$$
q_{j}\left(c_{j} \mid \mathbf{r}\right) \propto c_{j}^{\bar{c}_{j} / \beta_{j}-1} e^{-c_{j} / \beta_{j}}
$$

where $\mathbf{r}\left(\equiv r_{1}, r_{2}, \ldots\right)$ is a vector of spike counts, with $r_{i}(t)$ set to the number of spikes from ORN receptor type $i$ in a window of size $\Delta t$; in our simulations, we set $\Delta t$ to 50 ms. The parameters $\bar{c}_{j}$ and $\beta_{j}$ determine the approximate probability distribution over the concentration of odor $j$. These parameters have a natural interpretation: $\bar{c}_{j}$ is the mean concentration and $\bar{c}_{j} \beta_{j}$ is the variance. Both are important for inferring whether or not an odor is present. In particular, the lower the mean the less likely an odor is to be present, and for a fixed mean, the higher the variance the more likely the odor is to be present - because it's more likely that the true concentration is relatively high.

Note that we can't represent arbitrary posterior distributions over concentration; instead, our posterior distribution is summarized by two parameters. Moreover, although both are important, the second one, $\beta_{j}$, turns out to be independent of activity, $\mathbf{r}$, and only weakly dependent on odor, $j$ (see Online Methods, Eqs. (22b) and (38)). Thus, the distribution in Eq. (3) is reasonably well summarized by $\bar{c}_{j}$, and that's what we focus on. Not surprisingly, given the difficulty of the inference task, $\bar{c}_{j}$ depends on the activity of the ORNs, $\mathbf{r}$, in a relatively complicated way. However, they can be computed by the network shown in Fig. 1. Explicit equations describing the time evolution of the various cells in the network are given in Online Methods, Eq. (28). Here we provide a qualitative description.

Input to our network comes from the ORNs: each ORN receptor type projects, via a glomerulus (which we do not model), to one mitral cell; those cells then interact, via approximately reciprocal dendro-dendritic connections, with the granule cells. We ignore any spread of signals in the granule cells; essentially, each mitral cell has its own private connection. However, as is observed experimentally, ${ }^{13}$ activity at the soma of the granule cells is transmitted to its dendrites, and modulates activity there. The mitral cells also project, via the lateral olfactory tract, to the "mean concentration" cells in piriform cortex the cells labeled $\bar{c}_{j}$ in Fig. 1 . The mean concentration cells then project back to the granule cells, which in turn inhibit the mitral cells.

As this explanation suggests, the network implements a negative feedback loop: the mitral cells excite the mean concentration cells in piriform cortex, those cells feed back to the bulb and excite the granule cells, and the granule cells inhibit the mitral cells. That feedback loop acts iteratively: it infers a set of odors, compares that inference to the incoming information, uses the comparison to refine the inference, and then repeats the process. Here we illustrate this for an olfactory scene containing, for definiteness, three odors.

To simplify the analysis, we focus on mitral cells and mean concentration cells. The granule cells are of course critical to the operation of the network, but as shown in Online Methods (in particular Eq. (31)) their main effect is to provide divisive inhibition. For our network the specific form of the divisive inhibition involves a square root: the activity of the 


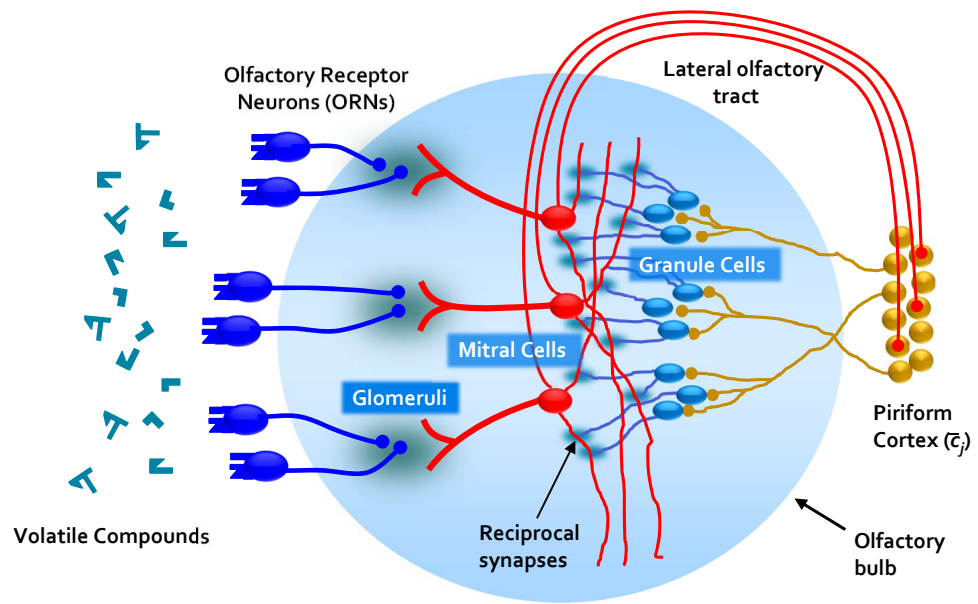

Figure 1: Circuit diagram. The olfactory receptor neurons (ORNs), which respond to volatile compounds in the air, are divided into different receptor types. All ORNs of the same receptor type project, via a glomerulus (which we do not model), onto a mitral cell (each of our mitral cells should be thought of as a "meta-mitral" cell, since in the real circuit there are approximately 15 mitral cells for every glomerulus). The mitral cells interact, via approximately reciprocal connections, with the granule cells (which are inhibitory and, in our network, outnumber the mitral cells by a factor of 3). The mitral cells also project, via the lateral olfactory tract, to the "mean concentration" cells in piriform cortex (labeled $\bar{c}_{j}$ ). These are the cells that carry direct information about the distribution over concentration for odor $j$. They also provide feedback - essentially an estimate of the mean concentration - to the granule cells: when the odors are successfully inferred, the feedback signal cancels, via the inhibitory granule cells, the feedforward drive from the ORNs, and the mitral cells are pushed toward their baseline firing rates.

$i^{\text {th }}$ mitral cell, $m_{i}$, is given approximately by

$$
m_{i} \propto \frac{\left(\gamma_{i} r_{i}\right)^{1 / 2}}{\left(\nu_{0, i}+\Delta t^{-1} \sum_{j} w_{i j} \bar{c}_{j}\right)^{1 / 2}}
$$


where $r_{i}$ is the activity of ORN receptor type $i$ and $\gamma_{i}$ is an unimportant scale factor (it cancels the factor of $\gamma_{i}^{-1}$ that appears in Eq. (5) below). The denominator in this equation is due to the granule cell feedback, which provides targeted divisive inhibition via the $w_{i j}-$ the same weights that generated the ORN responses (see Eq. (1)). Those weights combine connections from the mean concentration cells in piriform cortex to the granule cells (green axons in Fig. 1) with connections from granule cells to mitral cell dendrites (blue dendrites in Fig. 1).

The mitral cells drive the mean concentration cells, thus completing the feedback loop. As shown in Online Methods (in particular Eq. (28a)), the drive to mean concentration cell $i$ is linear in $m_{i}^{2}$,

$$
\tau_{c} \frac{d \bar{c}_{j}}{d t}+\bar{c}_{j}-\alpha_{0} \beta_{j} \propto \bar{c}_{j} \sum_{i} \gamma_{i}^{-1} m_{i}^{2} w_{i j}
$$

where $\tau_{c}$ is the time constant of the mean concentration cells and $\alpha_{0} \beta_{j}$ is the mean concentration associated with the prior. The main effect of the term $\tau d \bar{c}_{j} / d t$ is to introduce a delay, as it forces the mean concentration cells to integrate the drive from the mitral cells (a similar term should appear in Eq. (4), but it's less important, so to simplify the explanation we did not include it). Note that the mitral cells drive the mean concentration cells via the transpose of the weights, $w_{i j}$. Unlike the divisive inhibition associated with the granule cells, which involve several synapses, the mitral cells connect directly to the mean concentration cells, via the lateral olfactory tract shown in Fig. 1.

Immediately after odor onset, $\bar{c}_{j}$ is small - it's not far from $\alpha_{0} \beta_{j}$ (which is about 0.002 ). We can thus ignore the second term in the denominator of Eq. (4). Consequently, $m_{i} \propto r_{i}^{1 / 2}$, and mitral cells closely track ORN activity. This can be seen in Fig. 4a below, which shows ORN activity (top row of panel a) increasing linearly and mitral cell activity (second row) also increasing approximately linearly. The approximately linear increase of mitral cells does not last long, though; only about $20 \mathrm{~ms}$. However, that $20 \mathrm{~ms}$ is critical: Replacing $m_{i}^{2}$ by $\gamma_{i} r_{i}$ in Eq. (5), we see that the mean concentration cells in piriform cortex are driven by $\sum_{i} r_{i} w_{i j}$. This is an approximation to the template matching signal (see Fig. 2 caption and Online Methods, Sec. 6), and in the first $20 \mathrm{~ms}$ it provides the network's initial estimate of which odors are present. The quality of that estimate can be seen in Fig. 2b, which shows the activity of the mean concentration cells at $20 \mathrm{~ms}$. Even though only $20 \mathrm{~ms}$ has elapsed, the presented odors (blue) are mainly larger than the non-presented ones (red). More quantitatively, a threshold set to the smallest presented odor (black line) would produce only 21 false positives (out of 637 possible false-positives). This is much better than chance, indicating that the initial feedforward sweep of activity is beneficial. The activity at $20 \mathrm{~ms}$ is also qualitatively similar to the actual template matching signal (Fig. 2a), in the sense that a threshold set to the smallest presented odor (black line) would produce 15 false positives. (The lack of quantitative similarity between Figs. 2a and b is due primarily to nonlinearities in the network.)

Once inferred odor concentrations start to grow, their growth accelerates. That's because of the factor of $\bar{c}_{j}$ on the right hand side of Eq. (5). This creates a positive feedback loop, and thus leads to a "rich get richer" effect. But the rich do not get uniformly richer; there's also a consistency requirement. Because of the negative feedback, cells representing odors need appropriate drive from the ORNs to sustain their growth. This is a collective phenomenon, 


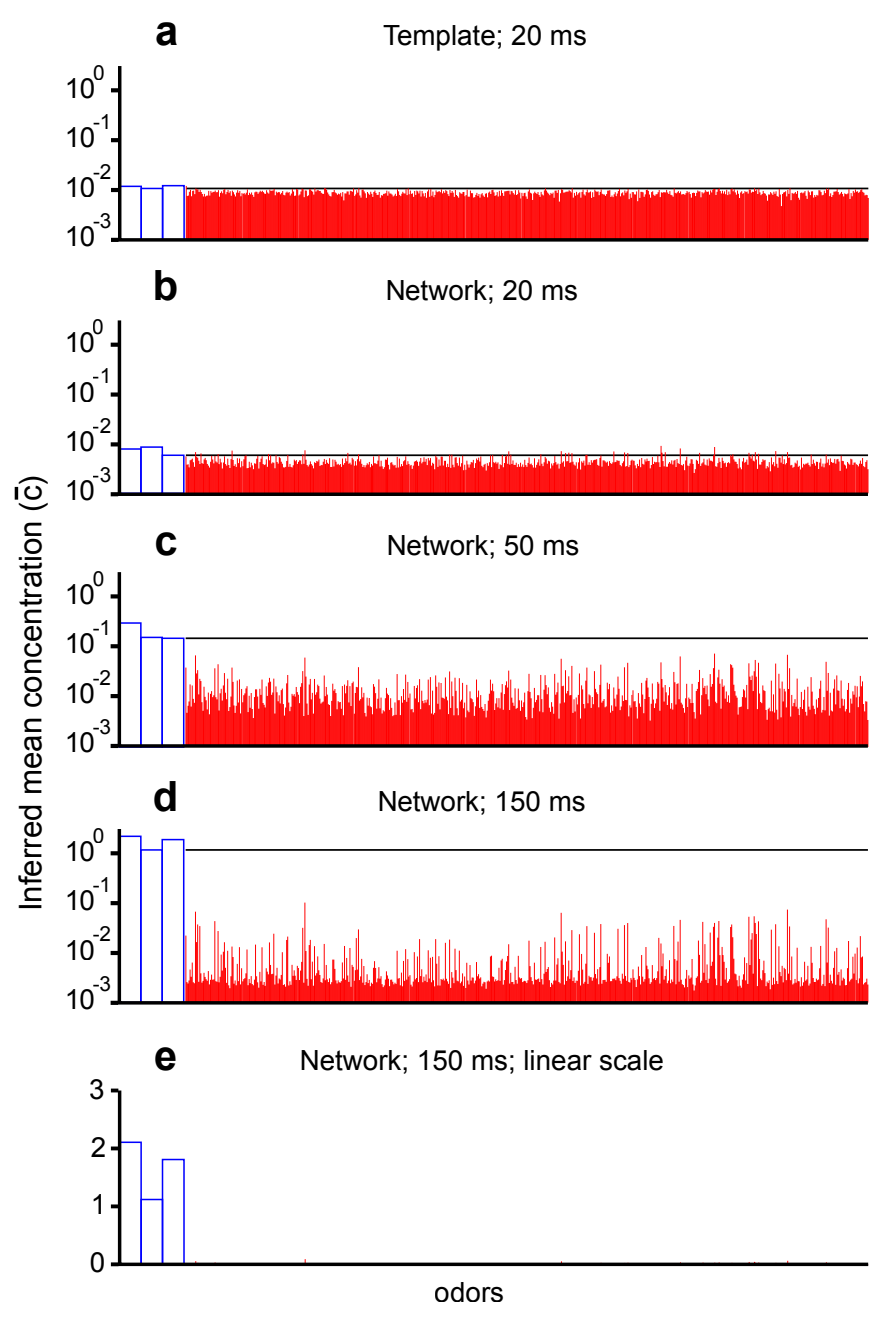

Figure 2: Evolution of the mean concentration cells (the $\bar{c}_{j}$ ) when three odors are presented. The first three odors (blue) correspond to the presented odors; the remaining 637 (red) correspond to the odors that were not presented. All plots except the one in panel e are on a log scale. a. Template matching estimate, $\sum_{i} r_{i} w_{i j} /\left(\sum_{i} w_{i j}^{2}\right)^{1 / 2} 20 \mathrm{~ms}$ after odor onset. b. Activity of the mean concentration cells $20 \mathrm{~ms}$ after odor onset. c. Activity $50 \mathrm{~ms}$ after odor onset. d. Activity $150 \mathrm{~ms}$ after odor onset. e. Activity $150 \mathrm{~ms}$ after odor onsets, but on a linear scale. 
which can be understood by considering the following example. Suppose $w_{i 1}$ is nonzero for $i=1, \ldots, 10$ and zero for all the other $i$, so odor 1 activates mitral cells $1-10$. If only odor 1 were present, ORNs 1-10 would be activated (see Eq. (1)), and they would drive mitral cells 1-10 (see Eq. (4)). Those ten mitral cells would then strongly drive the cell representing odor 1 (see Eq. (5)). They would, of course, also drive other cells, but less effectively, as only a fraction of the ten active mitral cells, not all of them, would affect the other cells. Because of the negative feedback, the cells representing the other odors would not be able to sustain their activity. Thus, $\bar{c}_{1}$ would grow, but the other inferred odor concentrations would stay near background.

In more realistic situations the idea is the same: an initial template matching signal causes the correct odor to have reasonably high activity; the rich get richer effect (the factor of $\bar{c}_{j}$ on the right hand side of Eq. (4)) causes odors with initially elevated activity to grow; and consistency ensures that the correct odors are the ones most likely to eventually reach relatively high activity. Note that in our network we use a prior that does not favor any particular odor, but it is easy to change the prior in an odor specific way simply by letting $\beta_{0}$ in Eq. (5) depend on odor, $j$; that is, let $\beta_{0} \rightarrow \beta_{0 j}$, with $\beta_{0 j}$ larger for odors that are more likely to appear.

The growth of the odors is illustrated in Fig. 2. We have already seen that the activity at $20 \mathrm{~ms}$ (panel b) provides a reasonable estimate of which odors are present. Only $50 \mathrm{~ms}$ later (panel c), the presented odors are starting to rise above the noise. In fact, a threshold set to the activity level of the smallest presented odor (black line) is a factor of more than two larger than the activity level of the largest non-presented odor. And at $150 \mathrm{~ms}$ (panel d on a log scale; panel e on a linear scale), the smallest presented odor is an order of magnitude larger than all of the non-presented odors (black line in panel d).

Although the correct odors were inferred, their mean concentrations were underestimated: the presented odors all had concentrations of 3, but the inferred mean concentrations were between 1 and 2 (Fig. 2e). This is a typical side effect of Bayesian inference, in which inferred quantities are biased toward the prior, which in our case favors low concentrations. ${ }^{14}$

In this example the activity of the mean concentration cells was either very high or very low, so it was clear which odors were present and which were not. However, had the activity been at an intermediate level it would have been less clear. To handle those cases, we need a mapping from the approximate posterior distribution (given in Eq. (3)) to the probability that an odor is present. Because, as discussed above, the approximate distribution is characterized primarily by the mean concentration, $\bar{c}_{j}$, we focus on the mapping from $\bar{c}_{j}$ to probability. To get that mapping, we performed many simulations, each with a different combination of odors, and combined them to compute the probability that an odor is present given $\bar{c}_{j}$ (see Online Methods, Sec. 5, for details). The resulting plot of probability versus $\bar{c}_{j}$ is shown in Fig. 3a. Animals can easily learn, from experience, an approximation to this relationship. In addition, as shown in Figs. $3 \mathrm{~b}$ and $\mathrm{c}$, the inferred mean concentration, $\bar{c}_{j}$, is well predicted, but slightly lower than, the true concentration, $c_{j}$. Thus, above a concentration of about 0.3-0.5 (depending on the number of presented odors) the inferred mean concentration, and thus the probability than an odor is present, is invariant to concentration. 
A
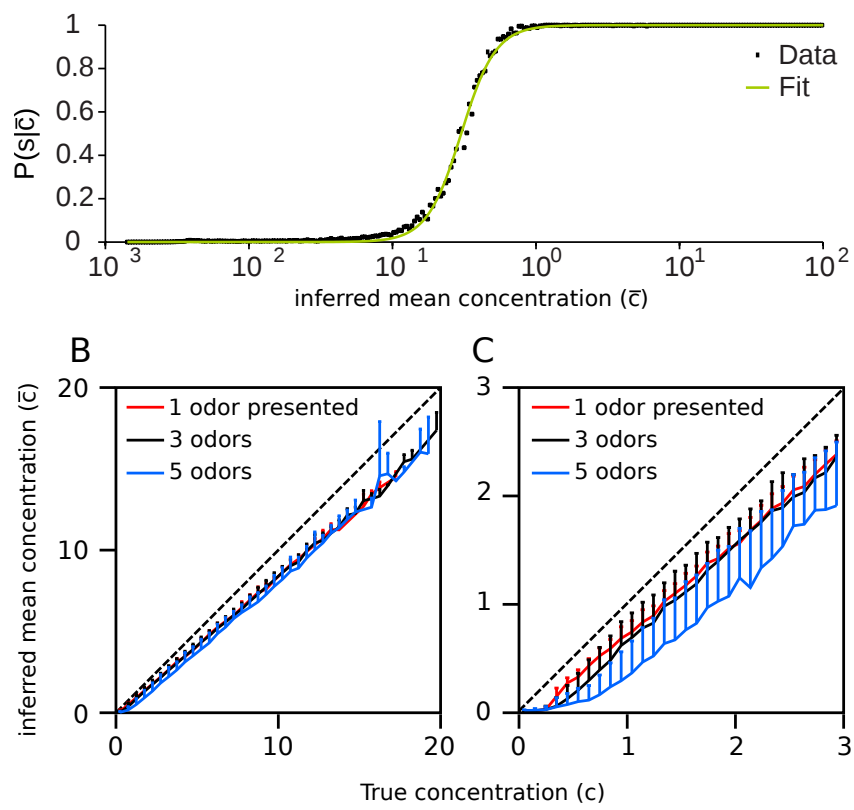

Figure 3: Probability of odor presence and inferred concentration. a. Probability that an odor is present given the inferred mean concentration, $\bar{c}$, computed by the network. b. Inferred mean concentration $\left(\bar{c}_{j}\right)$ versus true concentration $\left(c_{j}\right)$; error bars (shown only in the positive direction to reduce clutter) are standard deviations. On this scale, the inferred mean concentration is approximately proportional to (but slightly smaller than) the true concentration. c. Same as panel b, but for concentrations between 0 and 3. Especially at low concentrations, our network underestimates the true concentration, with the underestimate larger when more odors are presented. This is due to the sparse prior on odors, which introduces a bias toward low inferred concentration. All three panels were constructed by performing a large number of simulations, each with a different olfactory scene; see Online Methods, Sec. 5 . 


\section{Compatibility with known physiology}

Our circuit is, of course, simplified relative to the true one. In the true circuit, each ORN receptor type projects to two glomeruli (one on either side of the olfactory bulb); these in turn innervate mitral cells, with each glomerulus innervating about 15 mitral cells; and each mitral cell forms dendro-dendritic connections with about 30 granule cells. ${ }^{15}$ Signals can propagate within granule cells, and so inhibit other mitral cells. In addition to this main circuitry, there is lateral inhibition among the glomeruli. ${ }^{15}$ Finally, tufted and mitral cells have different response properties and projection patterns ${ }^{16,17}$ and connectivity to and from the olfactory bulb is not limited to the piriform cortex. ${ }^{18}$ In sum, we have bypassed the glomeruli, replaced the set of mitral and tufted cells by one meta-mitral cell, drastically reduced the number of granule cells, and ignored the spread of depolarization in those cells. It will be important to include this more complex circuitry in future work. However, our circuit does contain the main cell types, mitral cells and granule cells, it preserves the approximately reciprocal connections between those cell types, and it faithfully mirrors the main connections between the olfactory bulb and piriform cortex.

In the next several sections we investigate how this circuit responds to a range of olfactory scenes. For all simulations the network contains 160 ORN receptor types, 160 mitral cells, 480 granule cells, 640 "mean concentration" cells in piriform cortex (the cells labeled $\bar{c}_{j}$ in Fig. 1), and there are 640 possible odors (see Online Methods: Secs. 3 and 4 for additional details, and Table 1 for parameters).

\section{Response to a known odor}

We first tested our model in response to a single known odor. Such an odor was generated by setting the concentration of odor $1, c_{1}$, to 3 (the mean value of presented odors; see Eq. (2) and note that $\beta_{\text {prior }}=3$ ), and the concentrations of all the other odors to zero. Activity of our four cell types - ORNs, mitral cells, granule cells and mean concentration cells (the cells in piriform cortex that read out the odor) - are shown in Fig. 4a. The ORN activity quickly rises to a steady state, and stays there. The mitral cells, however, have more interesting dynamics: they exhibit an initial burst of activity from the ORN input, but that activity is terminated by inhibitory feedback from piriform cortex. In this example, the circuit correctly inferred which odor was present, so only one of the mean concentration cells (the correct one, $\bar{c}_{1}$ ) had appreciable activity.

The key observation is that when the odor is correctly inferred, activity in cortex mainly cancels the incoming ORN signal, and so mitral cell activity drops (as also predicted by Koulakov and Rinberg ${ }^{19}$ ). This is broadly consistent with data in awake behaving animals. $^{20-23}$

To show that the drop in mitral cell activity is due to feedback, we "cooled" cortex: we reduced the strength of the feedback connections from the mitral cells to the mean concentration cells by a factor of 100. The resulting activity, again for all four cell types, is shown in Fig. 4b. With activity in cortex nearly non-existent, granule cells have relatively low activity. Thus, mitral cell activity stays high, as it mainly represents the raw ORN signal. This is what is observed in rabbits when fibers from piriform cortex to the bulb are cut $^{24}$ and in mice when piriform cortex is pharmacologically suppressed. ${ }^{25}$ 

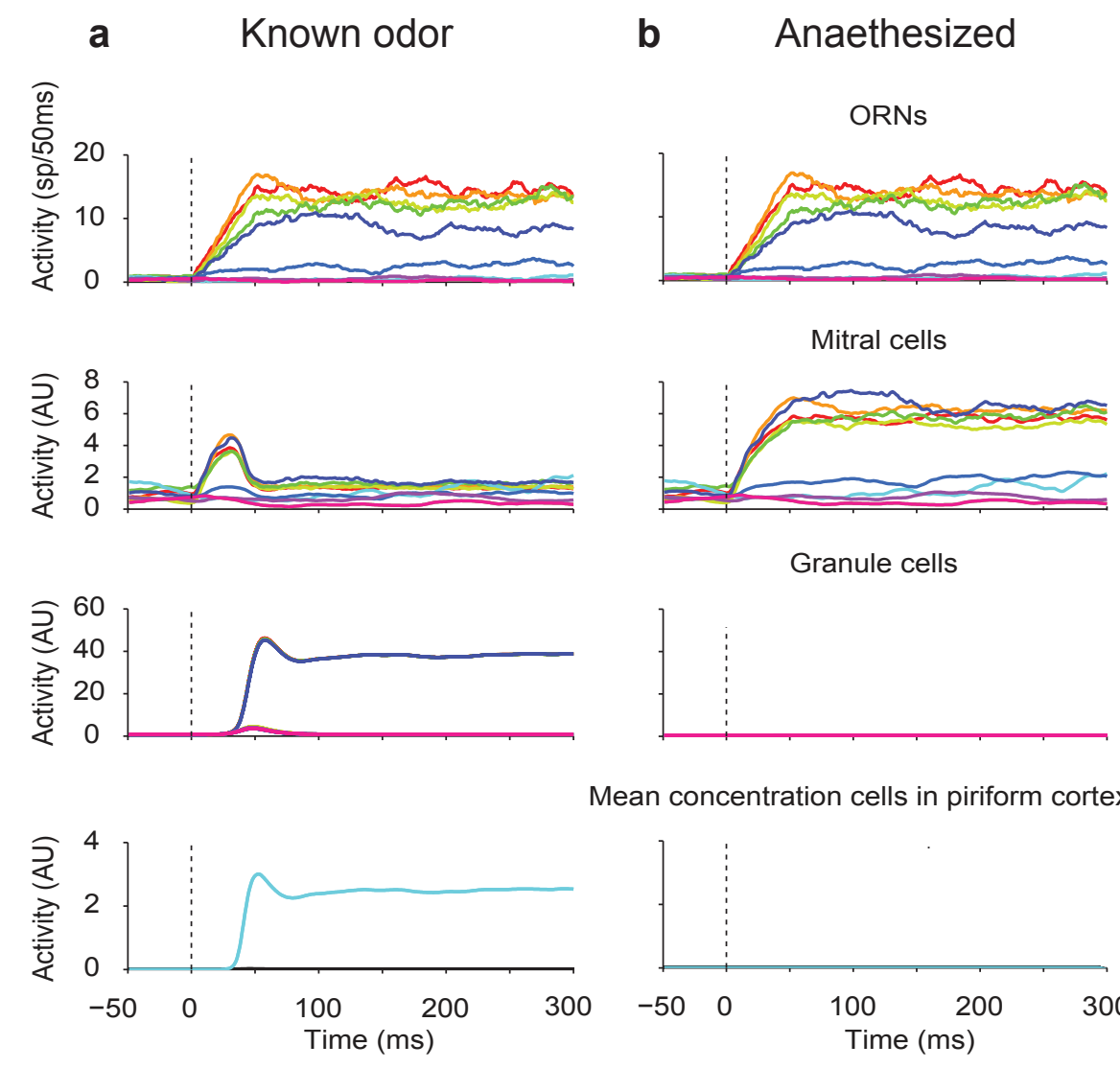

c Unknown odor
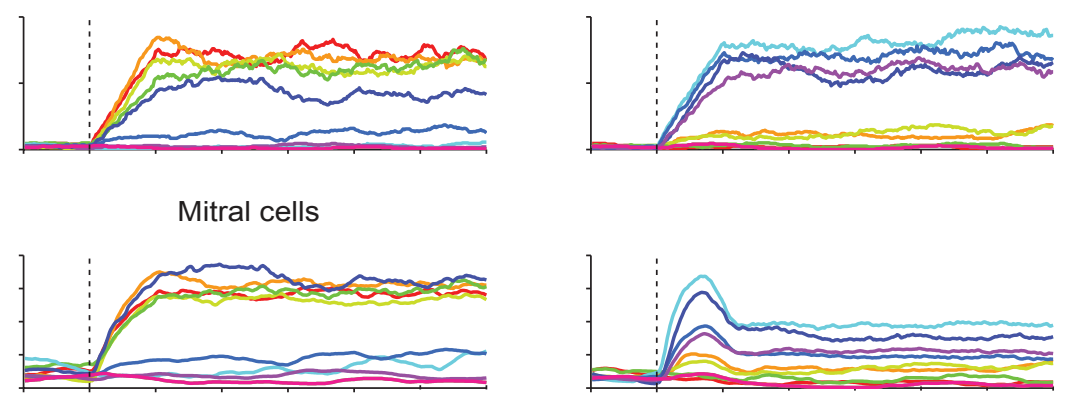

Granule cells
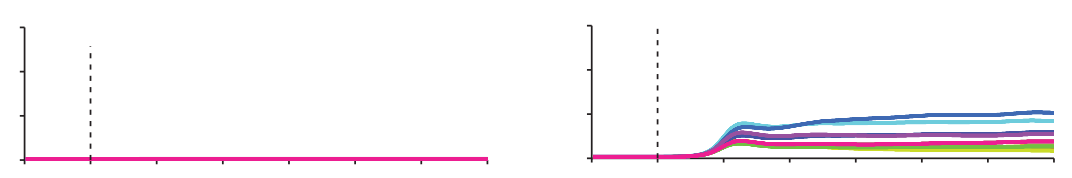

Mean concentration cells in piriform cortex

Figure 4: Activity in response to a single odor whose concentration is equal to 3 , the mean concentration of present odors. Top to bottom: activity of 8 ORN receptor types; activity of the 8 mitral cells that receive input from the ORNs in the top row, matched by color. activity of the 8 granule cells whose main input comes from the mitral cells in the second row, matched by color; activity of all 640 mean concentration cells in piriform cortex. ORNs: activity is the number of spikes in $50 \mathrm{~ms}$; Other cells: activity is in arbitrary units. The traces are an average over 10 trials. a. The odor is known to the animal. The cyan line in the bottom panel corresponds to the presented odor (odor 1); the (barely visible) black lines to all the other odors. b. The odor is known to the animal, but the feedback from the mitral cells to piriform cortex is reduced by a factor of 100 . Because there is almost no cortical activity, granule cells lose a large component of their excitatory input, and so have low activity - too low and indiscriminate to cancel mitral cell activity. c. The odor is unknown to the animal. The cortex tries to explain the ORN activity with many odors at low concentration, resulting in indiscriminate granule cell activity, and only partial cancellation of mitral cell activity. There is no cyan line, as the presented odor has not been learned. 


\section{Response to an unfamiliar odor}

A milder version of this lack of cancellation can be seen when an unfamiliar odor is presented; we mimic this by setting the mean firing rate of ORN receptor type $i$ to $\nu_{0, i}+\tilde{w}_{i} \beta_{\text {prior }}$ where the $\tilde{w}_{i}$ are a set of weights that don't correspond to any of the odors known to the animal, and, recall, $\beta_{\text {prior }}=3$. The resulting activity in the olfactory bulb and piriform cortex is shown in Fig. 4c. Because $\tilde{w}_{i}$ isn't known by the circuit, it can't infer which odor is present. Instead, piriform cortex tries to explain the ORN activity with many odors at low concentration. The activity in piriform cortex is sufficient to elicit granule cell activity; however, it's not the right activity, in the sense that it doesn't cancel the ORN input. Thus, although mitral cell activity is slightly lower than in Fig. 4b, it doesn't return to the values seen in Fig. 4a.

\section{Detecting an odor against a background}

One of the most common tasks faced by an animal is to detect a known odor against a background consisting of many other odors. Humans can do this reasonably well - they can detect a familiar odor in a background of 12 odors with about $65 \%$ accuracy. ${ }^{26}$ And, as shown by Rokni et al., ${ }^{2}$ mice can do even better: they can determine, with over $80 \%$ accuracy, whether or not a target odor is present against a background containing up to 13 distractor odors. We assessed the performance of our model on this task using the identical protocol as in the experiments of Rokni et al. Briefly, we presented between 0 and 14 background odors, with half the trials also containing a known target odor. The concentrations of all odors - both target and distractors - was the same, $c=3$. We reported the target present if its mean concentration $\left(\bar{c}_{j}\right)$ was above a threshold of 0.076 , and absent if it was below that threshold. In addition, we assumed that the mice licked spontaneously on $11 \%$ of the no-go trials. The value of the threshold for our network, and the spontaneous lick rate on no-go trials, were chosen to match as closely as possible the experimental results of Rokni et al.

The results are shown in Fig. 5. The solid black line is the fit to all trials; the solid blue and red lines are, respectively, fits to "Go" trials, corresponding to correct detection, and "NoGo" trials, corresponding to correct rejection. The dashed lines are the corresponding experimental fits of Rokni et al. ${ }^{2}$ Our simulations match well the experimental results, especially considering that we didn't specifically train our network on this task. In addition, good fits could be found for concentrations ranging from 0.3 to 5 , indicating that this result is relatively robust. We should point out, though, that for this task our inference algorithm may be overkill: performance matching that of the animals was achieved with a linear decoder. ${ }^{27}$ However, as we will see below, in general linear decoders do not do as well as our algorithm.

\section{Pattern decorrelation as a side effect of inference}

In a now seminal study, Friedrich and Laurent showed that average mitral cell activity in the decerebrate zebrafish decorrelates over time: the average activity associated with two odors can be very similar (highly correlated) at the beginning of a trial, but very different (uncorrelated) at the end of a trial. ${ }^{28}$ (Note that we are referring to so-called "pattern" correlation, which measures the similarity of average responses; see Fig. 6.) The conclusion was that the olfactory bulb actively separates odors, making them easier to decode as time goes on. ${ }^{28}$ 


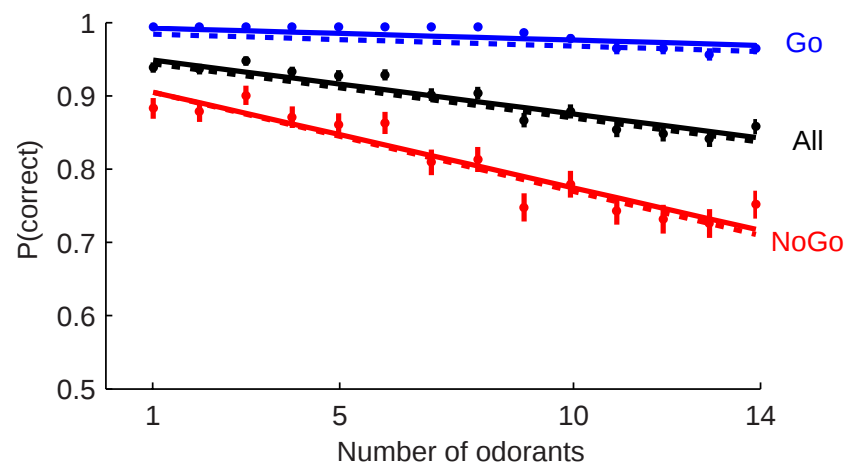

a

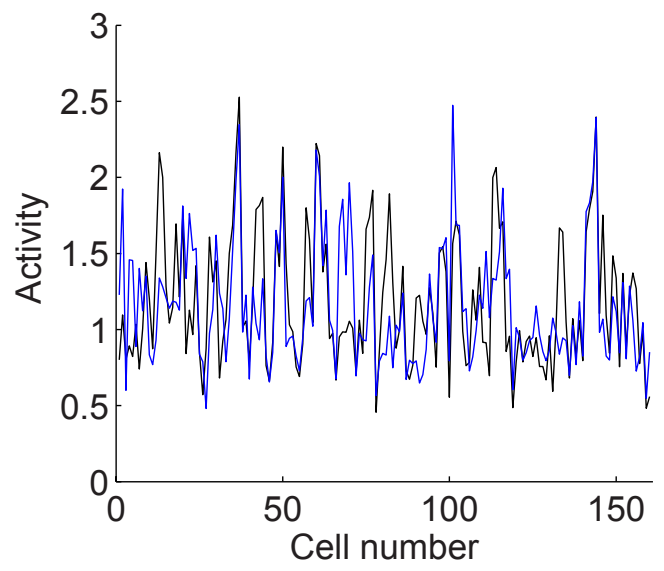

Figure 5: Performance of the model when detecting a known odor (target) against background odors. The target was present with probability $1 / 2$. The $y$-axis is the fraction of correct trials as a function of the number of odors present. Data are from our simulations, solid lines are least square fits, and dashed lines are fits to the experimental data of Rokni et al. ${ }^{2}$ Black: probability correct for all trials. Blue: probability correct for "Go" trials - trials containing the target. Red: probability correct for "NoGo" trials - trials not containing the target.

b

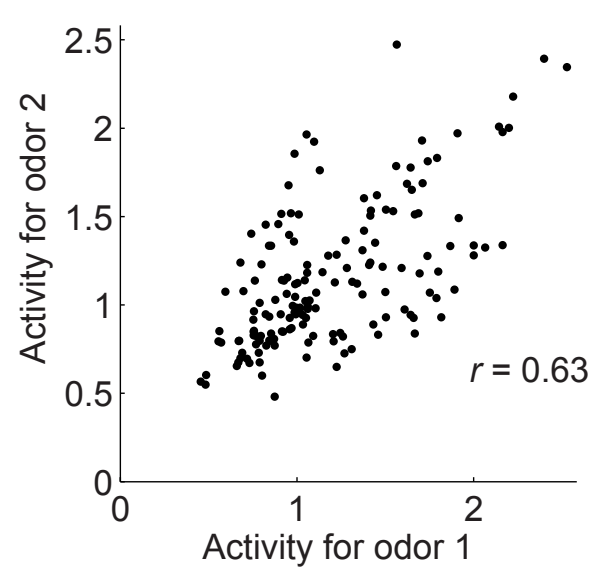

Figure 6: Pattern correlation as a measure of similarity between responses. a. Mean activity of 160 mitral cells (taken from our model) in response to two different odors (blue and black traces). b. Scatterplot of the mean activity of the 160 mitral cells in response to the same two odors. The pattern correlation, $r$ (defined to be the Pearson correlation coefficient for the scatterplot) is 0.72 , indicating that this pair of odors elicits relatively similar responses. 
To determine whether our model exhibits a similar pattern of decorrelation, we measured the average mitral cell responses to 10 randomly chosen odors - presented one at a time and computed, from 10 trials, the correlation coefficient between each pair of odors. This gave us a time series of $10 \times 10$ correlation matrices; snapshots of those matrices are shown in Fig. 7a. Some of the odors are initially strongly correlated, but over time their correlations weaken. Thus, odors that are initially very similar become less similar as time goes on - exactly what was seen by Friedrich and Laurent. ${ }^{28}$ However, other odors become more correlated with time - as also seen by Friedrich and Laurent. The net trend is that after odor onset the correlations decrease slightly, as indicated by the thick green line in Fig. 7b. This decrease was recently observed in mouse olfactory bulb mitral cells ${ }^{23}$ where, as in our simulations, the correlations dropped from about 0.4 to about 0.2 .

Critically, decorrelation in our model does not imply that odors are easier to distinguish as time goes on. In fact, it's the opposite: mitral cells contain less information, not more, as time goes on. That's because during inference, granule cells act to cancel the ORN input to the mitral cells. This is consistent with what has been reported in awake mice:21,29 decoding performance based on mitral cell activity increased immediately after odor onset, due to the arrival of information in the olfactory bulb, but decreased a short time later. It is inconsistent, however, with the study of Friedrich and Laurent in zebrafish, who found that decoding performance improved continually. ${ }^{28}$ The latter experiments were, however, performed in decerebrate fish, where cortical feedback does not operate normally. Moreover, the decorrelation took place over several seconds, whereas in rodents a single breathing cycle (200-300 ms) is often sufficient for odor discrimination. ${ }^{30}$ It is therefore unclear whether the results obtained in decerebrate fish are relevant for fast processing in behaving rodents. But for awake rodents, we are suggesting that decorrelation in the olfactory bulb is the consequence of the demixing performed by the olfactory bulb-piriform cortex loop, as opposed to a pattern separation mechanism.

\section{Comparison to other models}

Other demixing algorithms have been proposed; here we compare to two template based approaches. One is direct template matching, in which the concentration of an odor is given by the normalized dot product between the ORN responses and a template - a common approach in the olfactory system. ${ }^{31}$ The other is Fisher's linear discriminant, which is a sophisticated version of template matching: ORN responses are passed through a nonlinearity, and then distance to a template is computed using a metric that takes into account the pattern correlations. The latter was used recently in the analysis of a model of the fly olfactory system. ${ }^{32}$

We can compare these algorithms to ours using a generalization of receiver operating characteristic (ROC) curves: we plot hit rate (correct identification of odors) versus false positives as the threshold for detecting an odor changes. (Note that this analysis differs from that in Fig. 5, where the threshold was fixed.) Because more than one odor can be present at a time, we define the hit rate as the fraction of odors that were correctly identified. The results are shown in Fig. 8 (see Online Methods, Sec. 6, for additional information). As can be seen, our method does significantly better than the others. This is not surprising, as our method performs approximate Bayesian inference, whereas the other methods are more ad 
a
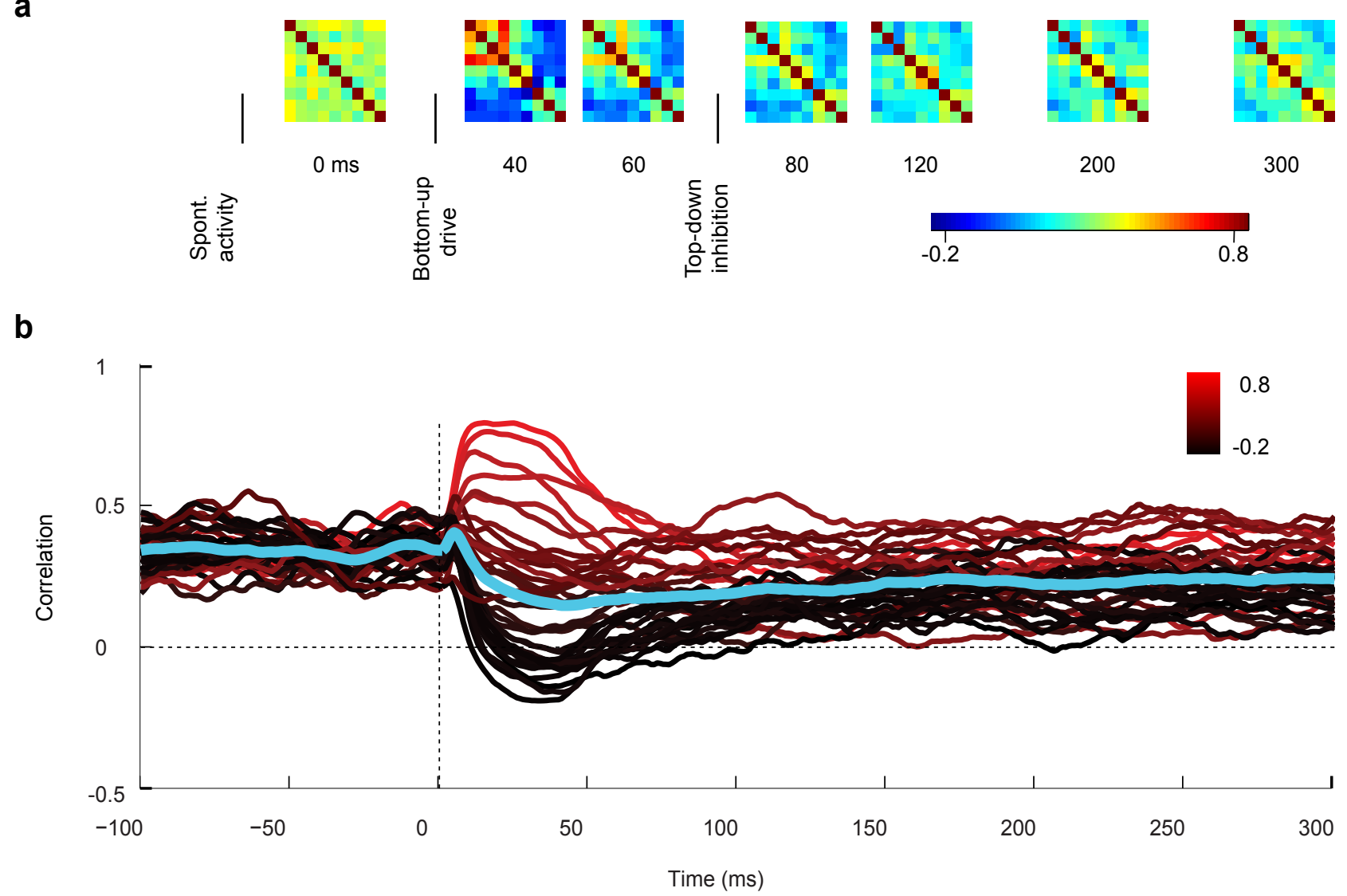

Figure 7: Evolution of pattern correlations in our model. a. Pattern correlation matrices (Pearson correlation coefficients; see Fig. 6) for mitral cells at several time points. At time $t=0$, before odor onset, activity is independent of odor; correlation coefficients are below 1 only because they were computed from a finite number of trials. In the early "bottom-up" phase $(t=40 \mathrm{~ms})$, correlations reflect odor similarity. However, in the later "top-down" phase $(t \geq 80 \mathrm{~ms})$, feedback from the cortex forces a return to baseline, which pushes correlations down slightly. Decorrelation occurs because of this shift, but only on average; there are many pairs that increase in correlation. Color indicates correlation coefficient. b. Same data as in the first panel, but plotted for all pairs of odors versus time. Color (black-red) indicates input pattern correlations (correlations among ORN responses); green is average correlation. 
a

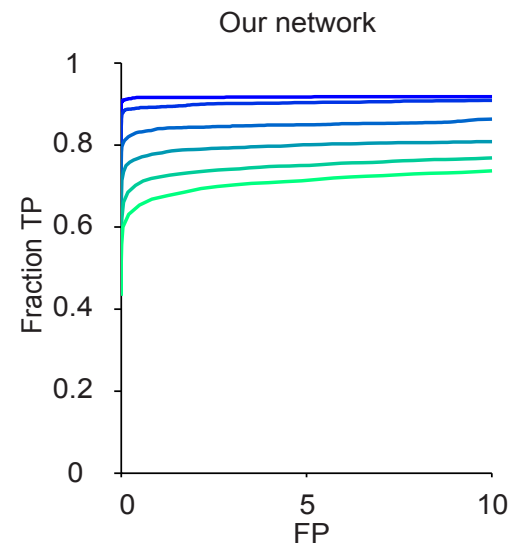

C

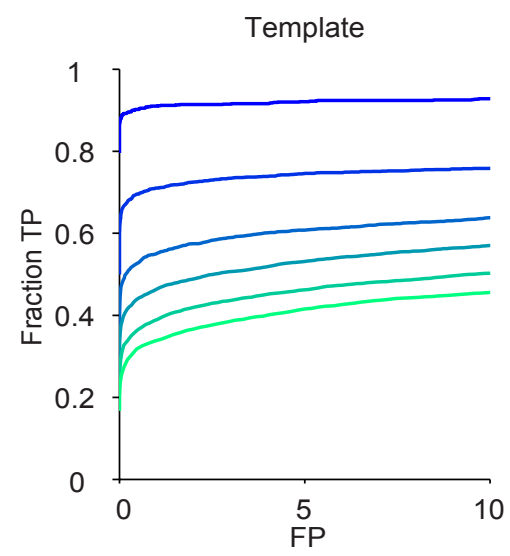

b

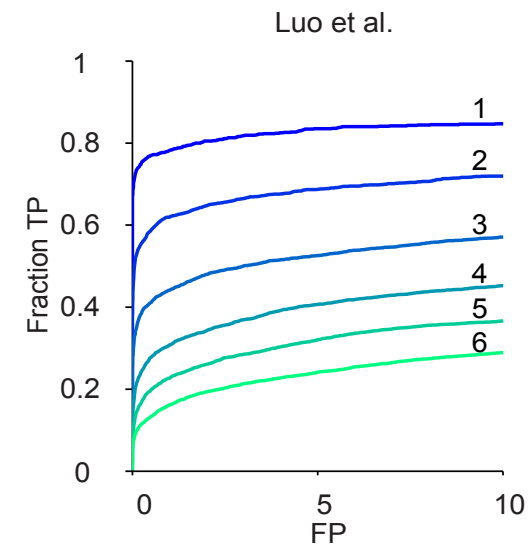

d

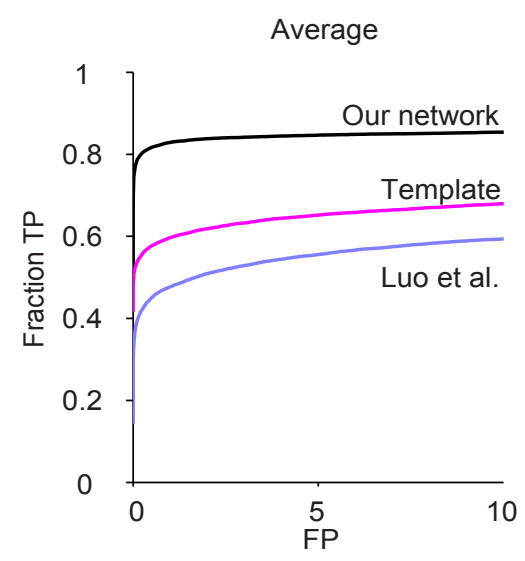

Figure 8: Generalized ROC curves (fraction of odors correctly identified versus number of false positives as the threshold varies); see text for details. In panels a-c, colors indicate number of odors present (panel b shows the mapping between color and number). a. Our network. b. Fisher's linear discriminant. c. Template matching. d. Average ROC curve, weighted by the probability that an odor is present, for the three methods.

hoc.

There are, of course, other approaches to demixing odors on a single sniff cycle. The vast majority of them effectively choose the odor concentrations that best match the observed firing rates, with a regularizing term that prevents overfitting. ${ }^{19,33,34}$ Our model falls into this class, with both the definition of "best match" and the regularizing term derived using approximate Bayesian inference (see Online Methods, Sec. 7).

\section{DISCUSSION}

\section{Treating olfaction as probabilistic inference}

The olfactory system, like all sensory systems, is faced with an inference problem: given the activity of the olfactory receptor neurons, the brain can never know exactly what odors are present. It can, though, do the next best thing: it can provide a probability distribution over odors. Given that distribution, it can make informed decisions ("do I eat that piece of fruit that smells like a grapefruit but looks like an orange?"). We thus treated olfaction as a probabilistic inference problem. For that we took the standard Bayesian approach: we wrote down an encoding model - a probabilistic transformation from odors to neural activity - and inverted that transformation using Bayes' theorem. As is typical of realistic problems, 
the second step could not be done exactly, so we had to compute an approximate posterior distribution over concentration.

The approximate inference algorithm we used matched what is seen in the early olfactory system in several ways. First, it led to circuitry that is broadly consistent with the anatomy of the olfactory bulb: we could naturally identify mitral cells and granule cells; those two cell types were reciprocally connected; the mitral cells projected to piriform cortex; and cells in piriform cortex projected back to granule cells. Second, our simulations produced, for a range of olfactory scenes and conditions, firing patterns that were consistent with in vivo activity. Third, activity patterns of the mitral cells evolved in a way consistent with pattern decorrelation. ${ }^{28}$ However, in our network pattern decorrelation is not synonymous with pattern separation, in the sense that it does not mean odors are easier to discriminate over time. Instead, decorrelation is a byproduct of our demixing algorithm.

\section{Previous work}

Ours is not, of course, the only model of the olfactory system; many have been proposed, with varying goals. ${ }^{5-9,19,33-47}$ Early theoretical work focused on reproducing the oscillations seen in the olfactory bulb in response to single odors, either with ${ }^{35,36}$ or without ${ }^{37-40}$ associative memory. Li was the first to address the problem of multiple odors, ${ }^{5,7}$ although she considered a situation in which odors are added on each sniff cycle. Hendin et al considered a one-sniff, one-odor version of this model, but with learning; ${ }^{41}$ later they extended it to allow the detection of one odor within a mixture. ${ }^{6}$ Other models that perform demixing on a single breathing cycle either assume that different odors have independent, and non-Gaussian, temporal fluctuations, ${ }^{8,9}$ or require a rather elaborate spike timing scheme; ${ }^{42,48}$ in both cases, it is unclear whether these assumptions are realistic. Thus, to our knowledge, our model is first one to identify a cortical-bulbar circuit that computes a probability distribution over odors, and does so in one breathing cycles without assuming independent fluctuations or relying on precise spike timing.

Other models have treated the olfactory bulb as a pre-processing step, one that makes it easier for downstream structures to infer what odor is present. ${ }^{19,33,34,43}$ Of these, the closest to our work are a set of models that both sparsify responses and reduce redundancy. ${ }^{19,33,34}$ In the hands of Koulakov and Rinberg, ${ }^{19}$ this was accomplished by reciprocal connections between mitral cells and granule cells. The other two studies ${ }^{33,34}$ adopted different implementations, but the underlying equations were very similar (see Online Methods, Sec. 7). Alternatively, some models use a form of "lateral inhibition," in which mitral cells inhibit each other locally, and inhibition is stronger between cells that are neighbors in olfactory space. ${ }^{44-46}$ Besides leading to sparse activity, these models tend to "decorrelate" responses, in the sense that responses to different odors become less similar. Finally, one model uses randomly connected recurrent networks to decorrelate responses, ${ }^{47}$ indicating that decorrelation doesn't need special circuitry. While these models result in redundancy reduction, it is not immediately clear how, or even whether, they help with odor segmentation. In particular, to our knowledge these model have not been used to demix a complex odor scene. 


\section{Experimental predictions}

Our model makes several testable predictions. First, inhibition of mitral cells by granule cells should be divisive (see Eq. (4)). Testing this prediction experimentally is difficult, as it requires knowledge of connectivity and whole cell in vivo recordings. However, it should be feasible in the not so distant future.

Second, our model predicts that when an unknown odor is presented, many granule cells should fire, but at low rates. In contrast, when a known odor is presented, a small fraction of granule cells should fire, but at relatively high rates. This could be assessed using standard methods, such as calcium imaging in awake animals; there is, in fact, already some evidence for it. ${ }^{49}$ Our model predicts essentially the same thing in piriform cortex: known odors should activate a relatively small fraction of neurons, but at high firing rates; an unknown odor, on the other hand, should activate a much larger fraction of neurons, but at much lower firing rate. This too could be assessed using calcium imaging.

$\mathrm{Li}$ and Hertz also predict different responses for known versus unknown odors. ${ }^{7}$ However, in their model those differences do not arise until after the first sniff cycle: the first sniff produces a strong response whether the odor is known, unknown, or a mixture of known and/or unknown odors; it is only on subsequent sniffs that the response in the bulb is suppressed, and then only if there is a single, known odor. Experimental tests of this prediction, which would go a long way toward distinguishing our model from that of Li and Hertz, ${ }^{7}$ would be relatively straightforward. (For additional experimental predictions of Li's model, many of which have been verified, see Ref. 50.)

\section{Broader implications}

The set of difficulties faced by the olfactory system is not unique to that system; it is common to all sensory modalities: we never observe objects in isolation, and the transformation from stimulus to neural response is noisy and often ambiguous. For example, we never observe visual objects against a uniform black background; instead, objects appear against complex backgrounds, and they are often partially occluded. Moreover, we see a two dimensional representation of the world from which we want to infer three dimensional shapes, a fundamentally ambiguous process. Thus, the lessons we learn from olfaction are likely to provide insight into other sensory modalities.

\section{ACKNOWLEDGMENTS}

Funding for AG-B and PEL was provided by the Gatsby Charitable Foundation; for ZFM and AP by the Human Frontiers Science Programme (RGP0027/2010) and the Simons Foundation (325057); for AP by the Swiss National Foundation (31003A_143707).

The authors declare no competing financial interests. 


\section{References}

[1] Chih-Ying Su, Karen Menuz, and John R. Carlson. Olfactory perception: Receptors, cells, and circuits. Cell, 139(1):45-59, 2009.

[2] Dan Rokni, Vivian Hemmelder, Vikrant Kapoor, and Venkatesh N. Murthy. An olfactory cocktail party: figure-ground segregation of odorants in rodents. Nature Neurosci., 17(9):1225-1232, 2014.

[3] B. Malnic, J. Hirono, T. Sato, and L. B. Buck. Combinatorial receptor codes for odors. Cell, 96(5):713-723, 1999.

[4] Alexandre Pouget, Jeffrey M. Beck, Wei Ji Ma, and Peter E. Latham. Probabilistic brains: knowns and unknowns. Nature Neurosci., 16(9):1170-1178, 2013.

[5] Z. Li. A model of olfactory adaptation and sensitivity enhancement in the olfactory bulb. Biological Cybernetics, 62(4):349-361, 1990.

[6] O. Hendin, D. Horn, and M. V. Tsodyks. Associative memory and segmentation in an oscillatory neural model of the olfactory bulb. J. Comput. Neurosci., 5(2):157-169, 1998.

[7] Z. Li and J. Hertz. Odour recognition and segmentation by a model olfactory bulb and cortex. Network: Computation in Neural Systems, 11(1):83-102, 2000.

[8] J. J. Hopfield. Olfactory computation and object perception. Proc. Natl. Acad. Sci., 88(15):6462-6466, 1991.

[9] O. Hendin, D. Horn, and J. J. Hopfield. Decomposition of a mixture of signals in a model of the olfactory bulb. Proc. Natl. Acad. Sci., 91(13):5942-5946, 1994.

[10] Timothy Connelly, Agnes Savigner, and Minghong Ma. Spontaneous and sensory-evoked activity in mouse olfactory sensory neurons with defined odorant receptors. J. Neurophys., 110(1):55-62, 2013.

[11] Jeff Beck, Katherine Heller, and Alexandre Pouget. Complex inference in neural circuits with probabilistic population codes and topic models. In Advances in Neural Information Processing Systems 25, 2012.

[12] Agnieszka Grabska-Barwińska, Jeff Beck, Alexandre Pouget, and Peter Latham. Demixing odors - fast inference in olfaction. In Advances in Neural Information Processing Systems 26, 2013.

[13] Veronica Egger, Karel Svoboda, and Zachary F. Mainen. Mechanisms of lateral inhibition in the olfactory bulb: efficiency and modulation of spike-evoked calcium influx into granule cells. J. Neurosci., 23(20):7551-7558, 2003.

[14] Yair Weiss, Eero P. Simoncelli, and Edward H. Adelson. Motion illusions as optimal percepts. Nature neuroscience, 5(6):598-604, 2002. 
[15] G.M. Shepherd, editor. The Synaptic Organization of the Brain. Oxford University Press, 5th edition, 2004.

[16] Izumi Fukunaga, Manuel Berning, Mihaly Kollo, Anja Schmaltz, and Andreas T. Schaefer. Two distinct channels of olfactory bulb output. Neuron, 75(2):320-329, 2012.

[17] Kei M. Igarashi, Nao Ieki, Myungho An, Yukie Yamaguchi, Shin Nagayama, Ko Kobayakawa, Reiko Kobayakawa, Manabu Tanifuji, Hitoshi Sakano, Wei R. Chen, and Kensaku Mori. Parallel mitral and tufted cell pathways route distinct odor information to different targets in the olfactory cortex. J. Neurosci., 32(23):797-7985, 2012.

[18] Hartwig Spors, Dinu Florin Albeanu, Venkatesh N. Murthy, Dmitry Rinberg, Naoshige Uchida, Matt Wachowiak, and Rainer W. Friedrich. Illuminating vertebrate olfactory processing. J. Neurosci., 32(41):14102-14108, 2012.

[19] A. A. Koulakov and D. Rinberg. Sparse incomplete representations: a potential role of olfactory granule cells. Neuron, 72(1):124-136, Oct 2011.

[20] R. A. Fuentes, M. I. Aguilar, M. L. Aylwin, and P. E. Maldonado. Neuronal activity of mitral-tufted cells in awake rats during passive and active odorant stimulation. $J$. Neurophys., 100(1):422-430, Jul 2008.

[21] Kevin Cury and Naoshige Uchida. Robust odor coding via inhalation-coupled transient activity in the mammalian olfactory bulb. Neuron, 68(3):570-585, 2010.

[22] Roman Shusterman, Matthew C. Smear, Alexei A. Koulakov, and Dmitry Rinberg. Precise olfactory responses tile the sniff cycle. Nature Neurosci., 14(8):1039-1044, July 2011.

[23] Olivier Gschwend, Nixon M. Abraham, Samuel Lagier, Frédéric Begnaud, Ivan Rodriguez, and Alan Carleton. Neuronal pattern separation in the olfactory bulb improves odor discrimination learning. Nature Neurosci., 18(10):1474-1482, 2015.

[24] D.G. Moulton. Electrical activity in the olfactory system of rabbits with indwelling electrodes. In Wenner-Gren Center International Symposium Series, volume 1, pages 71-84. Pergamon Press, Great Britain, 1963.

[25] G.H. Otazu, H. Chae, and D.F. Davis, M.B. Albeanu. Cortical feedback decorrelates olfactory bulb output in awake mice. Neuron., 86(6):1461-1477, 2015.

[26] A. Jinks and D. G. Laing. A limit in the processing of components in odour mixtures. Perception, 28(3):395-404, 1999.

[27] Alexander Mathis, Dan Rokni, Vikrant Kapoor, Matthias Bethge, and Venkatesh N. Murthy. Reading out olfactory receptors: Feedforward circuits detect odors in mixtures without demixing. Neuron, In press, 2016.

[28] Rainer W. Friedrich and Gilles Laurent. Dynamic Optimization of Odor Representations by Slow Temporal Patterning of Mitral Cell Activity. Science, 291(5505):889-894, 2001. 
[29] Olivier Gschwend, Jonathan Beroud, and Alan Carleton. Encoding odorant identity by spiking packets of rate-invariant neurons in awake mice. PLoS ONE, 7(1):e30155, 2012.

[30] Naoshige Uchida and Zachary F. Mainen. Speed and accuracy of olfactory discrimination in the rat. Nature Neurosci., 6(11):1224-1229, 2003.

[31] Kai Shen, Sina Tootoonian, and Gilles Laurent. Encoding of mixtures in a simple olfactory system. Neuron, 80(5):1246-1262, 2013.

[32] S. X. Luo, R. Axel, and L. F. Abbott. Generating sparse and selective third-order responses in the olfactory system of the fly. Proc. Natl. Acad. Sci., 107(23):1071310718, Jun 2010.

[33] Shaul Druckmann, Tao Hu, and Dmitri B. Chklovskii. A mechanistic model of early sensory processing based on subtracting sparse representations. In Advances in Neural Information Processing Systems 25, pages 1979-1987. Curran Associates, Inc., 2012.

[34] Sina Tootoonian and Mate Lengyel. A dual algorithm for olfactory computation in the locust brain. In Advances in Neural Information Processing Systems 27, pages 22762284. Curran Associates, Inc., 2014.

[35] B. Baird. Nonlinear dynamics of pattern formation and pattern recognition in rabbit olfactory bulb. Physica, 22D:150-175, 1986.

[36] P. Erdi, T. Gröbler, G. Barna, and K. Kaski. Dynamics of the olfactory bulb: bifurcations, learning, and memory. Biological Cybernetics, 69(1):57-66, 1993.

[37] W. J. Freeman. Nonlinear dynamics of paleocortex manifested in the olfactory EEG. Biological Cybernetics, 35(1):21-37, 1979.

[38] W. J. Freeman. EEG analysis gives model of neuronal template-matching mechanism for sensory search with olfactory bulb. Biological Cybernetics, 35(4):221-234, 1979.

[39] Z. Li and J.J. Hopfield. Modeling the olfactory bulb and its neural oscillatory processings. Biological Cybernetics, 61(5):379-392, 1989.

[40] Y Yao and W. J. Freeman. Model of biological pattern recognition with spatially chaotic dynamics. Neural Networks, 3:153-170, 1990.

[41] O. Hendin, D. Horn, and M. V. Tsodyks. The role of inhibition in an associative memory model of the olfactory bulb. J. Comput. Neurosci., 4(2):173-182, 1997.

[42] Carlos D. Brody and J. J. Hopfield. Simple networks for spike-timing-based computation, with application to olfactory processing. Neuron, 37(5):843-852, 2003.

[43] Davide Polese, Eugenio Martinelli, Santiago Marco, Corrado Di Natale, and Agustin Gutierrez-Galvez. Understanding odor information segregation in the olfactory bulb by means of mitral and tufted cells. PloS one, 9(10):e109716, 2014. 
[44] Armen C. Arevian, Vikrant Kapoor, and Nathaniel N. Urban. Activity-dependent gating of lateral inhibition in the mouse olfactory bulb. Nature Neurosci., 11(1):80-87, 2008.

[45] Thomas A. Cleland. Early transformations in odor representation. Trends in Neurosciences, 33(3):130-139, 2010.

[46] Thomas A. Cleland and Christiane Linster. On-Center/Inhibitory-Surround decorrelation via intraglomerular inhibition in the olfactory bulb glomerular layer. Frontiers in Integrative Neuroscience, 6, 2012.

[47] Martin T. Wiechert, Benjamin Judkewitz, Hermann Riecke, and Rainer W. Friedrich. Mechanisms of pattern decorrelation by recurrent neuronal circuits. Nature Neurosci., 13(8):1003-1010, 2010.

[48] J. J. Hopfield. Odor space and olfactory processing: collective algorithms and neural implementation. Proc. Natl. Acad. Sci., 96(22):12506-12511, 1999.

[49] H.K. Kato, M.W. Chu, J.S. Isaacson, and Takaki Komiyama. Dynamic sensory representations in the olfactory bulb: modulation by wakefulness and experience. Neuron, 76(5):962-975, 2012.

[50] Li Zhaoping. Olfactory object recognition, segmentation, adaptation, target seeking, and discrimination by the network of the olfactory bulb and cortex: computational model and experimental data. Current Opinion in Behavioral Sciences, 11:30-39, 2016. 


\section{Online methods}

\section{Introduction}

Here we provide details of our approximate inference algorithm, which leads ultimately to a set of equations that can be implemented in a neuronal network (Eq. (28)), with parameters given in Table 1. We then explain our method for determining the probability that an odor is present, how we compute ROC curves, and the relationship of our approach to other regularized models.

\section{Exact inference}

As in all Bayesian models, inference in olfaction requires that we invert the generative model and write down the probability distribution over concentration given spikes from the olfactory receptor neurons $(\mathrm{ORNs})$. We start by writing down the generative model and discussing exact inference.

The generative model consists of two parts: the likelihood, which is the probability of the data (in our case, spike trains from ORNs) given the odors that are present, and the prior probability distribution over odors. We already have an expression for the prior (Eq. (2)), so we just need the likelihood. In our model, ORNs generate spikes via a Poisson process. We assume that there is an effective time window, denoted $\Delta t$, for counting spikes; thus, the relevant quantity is the probability distribution over spike counts in that time window. Using $r_{i}$ to denote the spike count of ORNs of receptor type $i$ in time $\Delta t$, the distribution over $\mathbf{r}\left(\equiv r_{1}, r_{2}, \ldots, r_{N}\right)$ is given by

$$
p(\mathbf{r} \mid \mathbf{c})=\prod_{i=1}^{N} \frac{\left(\sum_{j=0}^{K} w_{i j} c_{j}\right)^{r_{i}}}{r_{i} !} e^{-\sum_{j=0}^{K} w_{i j} c_{j}}
$$

where $N$ is the number of ORN receptor types and $K$ is the number of odors (see Table 1 ). To take into account the background activity, the sum over $j$ runs from 0 to $K$ (instead of 1 to $K$, as it did in Eq. (1)), and we have defined

$$
w_{i 0} c_{0} \equiv \Delta t \nu_{0, i}
$$

The likelihood, Eq. (6), came from the encoding model given in Eq. (1) of the main text. In that encoding model, we assumed that ORNs respond linearly to log concentration. While this is a relatively common assumption, ${ }^{1-5}$ it is an approximation. It is certainly valid over one $\log$ unit, as individual ORNs are approximately linear over that range ${ }^{6}$ diversity within an ORN receptor type may extend that to two or three log units. ${ }^{7-9}$ A model taking into account nonlinearities would be interesting, but it is beyond the scope of this work. 
Combining Eq. (6) with the expression for the prior, Eq. (2), the distribution over concentration given spike count is

$$
p(\mathbf{c} \mid \mathbf{r}) \propto \prod_{i}\left(\sum_{j=0} w_{i j} c_{j}\right)^{r_{i}} e^{-\sum_{j=0} w_{i j} c_{j}} \prod_{j=1}\left[\left(1-p_{\text {prior }}\right) \delta\left(c_{j}\right)+p_{\text {prior }} \frac{e^{-c_{j} / \beta_{\text {prior }}}}{\beta_{\text {prior }}}\right] .
$$

While this expression is exact, it contains too much information to be useful to an organism; typically what an organism wants to know is whether or not a particular odor is present, not the full posterior distribution over all possible combinations of odors. In addition, the delta functions - which put point masses at zero concentration - complicates analysis. For instance, we can't find the most likely set of odors (the maximum a posteriori, or MAP, solution), as is sometimes done for complicated posteriors, because the delta functions mean zero concentration has infinite probability density. We assume, then, that the quantity of interest is the marginal distribution over each odor; for odor $j$, that distribution is given by

$$
p\left(c_{j} \mid \mathbf{r}\right)=\int d c_{1} d c_{2} \ldots d c_{j-1} d c_{j+1} \ldots d c_{K} p(\mathbf{c} \mid \mathbf{r}) .
$$

Unfortunately, it's not possible to perform that integral exactly. And even if we could perform the integral over the continuous part of the posterior distribution, because of the delta functions the number of integrals that have to be performed is exponential in the number of odors. We must, therefore, resort to approximate inference.

\section{Approximate inference}

There are two parts to our approximate inference algorithm. The first is to replace the prior - which is of the "spike and slab" form ${ }^{10}$ notoriously hard to analyze - with a smoother, approximate prior, denoted $p_{\text {approx }}(\mathbf{c})$. For that we use a product of Gamma distributions,

$$
p_{\text {approx }}(\mathbf{c})=\prod_{j=1} \frac{c_{j}^{\alpha_{0}-1} e^{-c_{j} / \beta_{0}}}{\Gamma\left(\alpha_{0}\right) \beta_{0}^{\alpha_{0}}}
$$

where $\Gamma\left(\alpha_{0}\right)$ is the standard Gamma function. This results in an approximate posterior, denoted $p_{\text {approx }}(\mathbf{c} \mid \mathbf{r})$,

$$
p_{\text {approx }}(\mathbf{c} \mid \mathbf{r}) \propto \prod_{i}\left(\sum_{j=0} w_{i j} c_{j}\right)^{r_{i}} e^{-\sum_{j=0} w_{i j} c_{j}} \prod_{j=1} c_{j}^{\alpha_{0}-1} e^{-c_{j} / \beta_{0}} .
$$

We follow a convention in which probabilities that depend only on $\mathbf{c}$ correspond to priors, whereas those that depend on $\mathbf{c} \mid \mathbf{r}$ correspond to posteriors.

We use $\alpha_{0}=1 / 3$, for reasons discussed below (see the text following Eq. (54) in Sec. 7 below). To ensure that the mean concentration of each odor under the approximate prior $\left(\alpha_{0} \beta_{0}\right)$ is the same as the mean under the exact prior in Eq. (2) $\left(p_{\text {prior }} \beta_{\text {prior }}\right)$, we chose $\beta_{0}$ to satisfy $\alpha_{0} \beta_{0}=p_{\text {prior }} \beta_{\text {prior. }}$. From Table $1, \beta_{\text {prior }}=3$ and $p_{\text {prior }}=3 / K$ where $K$ is the number of odors; thus, $\beta_{0}=27 / K$. 
Although this posterior is simpler than the true one, inference is still intractable, in the sense that even if we replaced $p(\mathbf{c} \mid \mathbf{r})$ with $p_{\text {approx }}(\mathbf{c} \mid \mathbf{r})$ on the right hand side of Eq. (9), we still couldn't do the integral. We could do MAP inference, but then we would lose any notion of uncertainty. Thus, the second part of our approximation is to use a variational approach: we find a parametrized distribution that is as close as possible to $p_{\text {approx }}(\mathbf{c} \mid \mathbf{r})$, as assessed by the Kullback-Leibler divergence. ${ }^{11}$ That distribution, denoted $q_{\text {var }}(\mathbf{c} \mid \mathbf{r})$, minimizes the KL divergence between $q_{\mathrm{var}}(\mathbf{c} \mid \mathbf{r})$ and $p_{\text {approx }}(\mathbf{c} \mid \mathbf{r})$, which is given by

$$
D_{K L}\left(q_{\mathrm{var}}(\mathbf{c} \mid \mathbf{r}) \| p_{\text {approx }}(\mathbf{c} \mid \mathbf{r})\right)=\int d \mathbf{c} q_{\mathrm{var}}(\mathbf{c} \mid \mathbf{r}) \log \frac{q_{\mathrm{var}}(\mathbf{c} \mid \mathbf{r})}{p_{\text {approx }}(\mathbf{c} \mid \mathbf{r})} .
$$

It turns out that directly minimizing the KL divergence is also hard, mainly because the first term on the right hand side of Eq. (11), $\left(\sum_{j} w_{i j} c_{j}\right)^{r_{i}}$, consists of products of a sum whenever $r_{i} \geq 2$. We can, though, minimize a bound on the KL divergence. To find the bound, we first use the multinomial theorem to write

$$
\left(\sum_{j=0} w_{i j} c_{j}\right)^{r_{i}}=\sum_{\mathbf{N}} \Delta\left(r_{i}-\sum_{j=0} N_{i j}\right) \prod_{j=0} \frac{\left(w_{i j} c_{j}\right)^{N_{i j}}}{N_{i j} !}
$$

where the sum over $\mathbf{N}$ is a sum over all sets of non-negative integers $N_{i j}$ such that the $N_{i j}$ add to $r_{i}$, a restriction that is enforced by the Kronecker delta,

$$
\Delta(n) \equiv \begin{cases}1 & n=0 \\ 0 & n \neq 0\end{cases}
$$

Inserting Eq. (13) into (11), we have

$$
p_{\text {approx }}(\mathbf{c} \mid \mathbf{r})=\sum_{\mathbf{N}} p_{\text {approx }}(\mathbf{c}, \mathbf{N} \mid \mathbf{r})
$$

where

$$
p_{\text {approx }}(\mathbf{c}, \mathbf{N} \mid \mathbf{r}) \propto \prod_{i}\left[\Delta\left(r_{i}-\sum_{j=0} N_{i j}\right) \prod_{j=0} \frac{\left(w_{i j} c_{j}\right)^{N_{i j}}}{N_{i j} !} e^{-w_{i j} c_{j}}\right] \prod_{j=1} c_{j}^{\alpha_{0}-1} e^{-c_{j} / \beta_{0}}
$$

To proceed, we introduce a second variational distribution, $q_{\mathrm{var}}(\mathbf{N} \mid \mathbf{r})$. It is straightforward to show that regardless of what this new variational distribution is, we can bound the KullbackLeibler divergence via

$$
D_{K L}\left(q_{\mathrm{var}}(\mathbf{c} \mid \mathbf{r}) \| p_{\text {approx }}(\mathbf{c} \mid \mathbf{r})\right) \leq \sum_{\mathbf{N}} \int d \mathbf{c} q_{\mathrm{var}}(\mathbf{c} \mid \mathbf{r}) q_{\mathrm{var}}(\mathbf{N} \mid \mathbf{r}) \log \frac{q_{\mathrm{var}}(\mathbf{c} \mid \mathbf{r}) q_{\mathrm{var}}(\mathbf{N} \mid \mathbf{r})}{p_{\text {approx }}(\mathbf{c}, \mathbf{N} \mid \mathbf{r})}
$$

Rather than minimizing the true KL divergence with respect to $q_{\mathrm{var}}(\mathbf{c} \mid \mathbf{r})$, we minimize the right hand side of Eq. (17) - a bound on the true KL divergence - with respect to $q_{\mathrm{var}}(\mathbf{c} \mid \mathbf{r})$ and $q_{\mathrm{var}}(\mathbf{N} \mid \mathbf{r})$. To do that, we differentiate with respect to $q_{\mathrm{var}}(\mathbf{c} \mid \mathbf{r})$ and $q_{\mathrm{var}}(\mathbf{N} \mid \mathbf{r})$ and set the resulting expressions to zero; this gives us

$$
\begin{aligned}
\log q_{\mathrm{var}}(\mathbf{c} \mid \mathbf{r}) & \sim \sum_{\mathbf{N}} q_{\mathrm{var}}(\mathbf{N} \mid \mathbf{r}) \log p_{\text {approx }}(\mathbf{c}, \mathbf{N} \mid \mathbf{r}) \\
\log q_{\mathrm{var}}(\mathbf{N} \mid \mathbf{r}) & \sim \int d \mathbf{c} q_{\mathrm{var}}(\mathbf{c} \mid \mathbf{r}) \log p_{\text {approx }}(\mathbf{c}, \mathbf{N} \mid \mathbf{r})
\end{aligned}
$$


where here, and in what follows, $\sim$ indicates equality up to an additive constant. To complete these equations, we need an expression for the log of the approximate posterior, $p_{\text {approx }}(\mathbf{c}, \mathbf{N} \mid \mathbf{r})$. Using Eq. (16), that's given by

$$
\begin{aligned}
\log p_{\text {approx }}(\mathbf{c}, \mathbf{N} \mid \mathbf{r}) & \sim \sum_{i}\left[\log \Delta\left(r_{i}-\sum_{j=0} N_{i j}\right)+\sum_{j=0} N_{i j} \log \left(w_{i j} c_{j}\right)-w_{i j} c_{j}-\log \left(N_{i j} !\right)\right] \\
& +\sum_{j=1}\left(\alpha_{0}-1\right) \log c_{j}-c_{j} / \beta_{0}
\end{aligned}
$$

Inserting this into Eq. (18) yields

$$
\begin{aligned}
\log q_{\mathrm{var}}(\mathbf{c} \mid \mathbf{r}) & \sim \sum_{j=1}\left[\left(\alpha_{0}-1+\sum_{i}\left\langle N_{i j}\right\rangle\right) \log c_{j}-\left(\sum_{i} w_{i j}+\frac{1}{\beta_{0}}\right) c_{j}\right] \\
\log q_{\mathrm{var}}(\mathbf{N} \mid \mathbf{r}) & \sim \sum_{i}\left[\log \Delta\left(r_{i}-\sum_{j=0} N_{i j}\right)+\sum_{j=0} N_{i j}\left\langle\log \left(w_{i j} c_{j}\right)\right\rangle-\log \left(N_{i j} !\right)\right]
\end{aligned}
$$

where the angle brackets indicate an average with respect to either $q_{\mathrm{var}}(\mathbf{c} \mid \mathbf{r})$ or $q_{\mathrm{var}}(\mathbf{N} \mid \mathbf{r})$, whichever is appropriate.

Examining these expressions, we see that $q_{\mathrm{var}}(\mathbf{c} \mid \mathbf{r})$ is Gamma distributed and $q_{\mathrm{var}}(\mathbf{N} \mid \mathbf{r})$ is multinomial distributed,

$$
\begin{aligned}
q_{\mathrm{var}}(\mathbf{c} \mid \mathbf{r}) & =\prod_{j=1} \frac{c_{j}^{\bar{c}_{j} / \beta_{j}-1} e^{-c_{j} / \beta_{j}}}{\Gamma\left(\bar{c}_{j} / \beta_{j}\right) \beta_{j}^{\bar{c}_{j} / \beta_{j}}} \\
q_{\mathrm{var}}(\mathbf{N} \mid \mathbf{r}) & =\prod_{i} r_{i} ! \Delta\left(r_{i}-\sum_{j=0} N_{i j}\right) \prod_{j=0} \frac{p_{i j}^{N_{i j}}}{N_{i j} !}
\end{aligned}
$$

where

$$
\begin{aligned}
\bar{c}_{j} & \equiv \beta_{j} \alpha_{0}+\beta_{j} \sum_{i}\left\langle N_{i j}\right\rangle \\
\beta_{j} & \equiv \frac{\beta_{0}}{1+\beta_{0} \sum_{i} w_{i j}} \\
p_{i j} & \equiv \frac{e^{\left\langle\log \left(w_{i j} c_{j}\right)\right\rangle}}{\sum_{j=0} e^{\left\langle\log \left(w_{i j} c_{j}\right)\right\rangle}} .
\end{aligned}
$$

Note that Eq. (21a) is the expression that appears in Eq. (3) of the main text (except that in the main text we do not include the normalization or the subscript "var"). Using Eq. (21), the averages in Eq. (22) are readily computed,

$$
\begin{aligned}
\left\langle N_{i j}\right\rangle & =r_{i} p_{i j} \\
e^{\left\langle\log \left(w_{i j} c_{j}\right)\right\rangle} & = \begin{cases}\Delta t \nu_{0, i} & j=0 \\
w_{i j} \beta_{j} e^{\psi\left(\bar{c}_{j} / \beta_{j}\right)} & j>0\end{cases}
\end{aligned}
$$


where $\psi$ is the digamma function,

$$
\psi(\alpha) \equiv \frac{d \log \Gamma(\alpha)}{d \alpha}
$$

Finally, inserting Eqs. (23a), (23b) and (22c) into (22a), we arrive at the update equation for $\bar{c}_{j}($ with $j \geq 1$,

$$
\bar{c}_{j}=\beta_{j} \alpha_{0}+\beta_{j} \sum_{i} \frac{r_{i} w_{i j} F_{j}\left(\bar{c}_{j}\right)}{\Delta t \nu_{0, i}+\sum_{k} w_{i k} F_{k}\left(\bar{c}_{k}\right)}
$$

where

$$
F_{j}\left(\bar{c}_{j}\right) \equiv \beta_{j} e^{\psi\left(\bar{c}_{j} / \beta_{j}\right)} .
$$

The function $F_{j}\left(\bar{c}_{j}\right)$ has a relatively simple dependence on $\bar{c}_{j}$ and $\beta_{j}$. Using the fact that $e^{\psi(x)} \approx x^{2} / 2$ if $x<1$ and $x-1 / 2$ if $x \geq 1$, we have

$$
F_{j}\left(\bar{c}_{j}\right) \approx \begin{cases}\bar{c}_{j}^{2} / 2 \beta_{j} & \bar{c}_{j}<\beta_{j} \\ \bar{c}_{j}-\beta_{j} / 2 & \bar{c}_{j} \geq \beta_{j} .\end{cases}
$$

Equation (25) is appropriate for simulations on a digital computer. However, because we are ultimately interested in a model of the mammalian olfactory system, we need a set of equations that is approximately consistent with known anatomy and physiology. And, of course, those equations must have the same fixed point (or points) as (25). Finding such a set of equations is a bit of an art, as they are not unique. The ones we found are

$$
\begin{aligned}
\tau_{c} \frac{d \bar{c}_{j}}{d t} & =\beta_{j} \alpha_{0}-\bar{c}_{j}+\beta_{j} F_{j}\left(\bar{c}_{j}\right) \sum_{i} \gamma_{i}^{-1} m_{i}^{2} w_{i j} \\
\tau_{m} \frac{d m_{i}}{d t} & =-m_{i}^{2} \Delta t \nu_{0, i}+\gamma_{i} r_{i}-m_{i} \sum_{k} w_{i k}^{m g} g_{i k} \\
\tau_{g} \frac{d g_{i k}}{d t} & =-g_{i k}+g_{k} w_{k i}^{g m} m_{i} \\
\tau_{g} \frac{d g_{k}}{d t} & =-g_{k}+\sum_{j} A_{k j} F_{j}\left(\bar{c}_{j}\right)
\end{aligned}
$$

where the $\gamma_{i}$ 's are arbitrary positive constants and the weights, $\mathbf{w}^{m g}, \mathbf{w}^{g m}$ and $\mathbf{A}$, satisfy

$$
w_{i j}=\sum_{k} w_{i k}^{m g} w_{k i}^{g m} A_{k j}
$$

In these equations we interpret $g_{k}$ as the activity of the soma of granule cell $k$ and $g_{i k}$ as the activity of spine $i$ associated with granule cell $k$. Dendro-dendritic connections are made at the spines.

To determine the initial conditions, we first ran the model for 2 seconds with all concentrations set to zero, and recorded the values of all the dynamical variables $\left(\bar{c}_{j}, m_{i}, g_{i k}\right.$ and 
$\left.g_{k}\right)$. Then, at the start of each simulation we took the recorded values and added to each of them Gaussian noise with standard deviation set to $10 \%$ of their value.

To show that in steady state - when all time derivatives are zero - these equations reduce to Eq. (25), we first use the fact that in steady state, $g_{i k}=g_{k} w_{k i}^{g m} m_{i}$ (Eq. (28c)). Inserting that into Eq. (28b) gives, again in steady state,

$$
m_{i}^{2}=\frac{\gamma_{i} r_{i}}{\Delta t \nu_{0, i}+\sum_{k} w_{i k}^{m g} w_{k i}^{g m} g_{k}}
$$

Thus, in our model granule cells are divisive. Using the steady state solution to Eq. (28d) to express $g_{k}$ in terms of the $\bar{c}_{j}$, and inserting the resulting expression into Eq. (28a) we arrive at Eq. (25).

In the main text (Sec. "A probabilistic model of olfaction"), we describe how our network performs inference. The basis for that description is the behavior of the mitral cells, the $m_{i}$ and the mean concentration cells, the $\bar{c}_{j}$. For the mitral cells we need only the steady state behavior; for that we simply insert Eq. (28d) into (30), yielding

$$
\gamma_{i}^{-1} m_{i}^{2}=\frac{r_{i}}{\Delta t \nu_{0, i}+\sum_{k} w_{i k} F_{j}\left(\bar{c}_{j}\right)}
$$

That corresponds to Eq. (4) of the main text, except that in the main text we approximated $F_{j}\left(\bar{c}_{j}\right)$ by $\bar{c}_{j}$, an approximation that is valid so long as $\bar{c}_{j}$ is sufficiently large (see Eq. (27)). For the mean concentration cells we need the time evolution, for which we can use Eq. (28a) directly. That correspond to Eq. (5) of the main text, except that again we approximated $F_{j}\left(\bar{c}_{j}\right)$ by $\bar{c}_{j}$.

\section{Parameters}

All parameters except the weights are given in Table 1. There are three sets of weights we need to specify: $w_{i k}^{m g}$ (granule cell $\rightarrow$ mitral cell), $w_{i k}^{g m}$ (mitral cell $\rightarrow$ granule cell), $A_{i k}$ (piriform cortex $\rightarrow$ granule cell). A diagram showing a qualitative picture of the weights is given in Fig. 1 of the main text. In words: there are three main granule cells associated with each mitral cell; for those the probability of a reciprocal connection is 1 . In addition, there are three secondary mitral cells on either side of it; for those the probability of a reciprocal connection is $1 / 2$. Feedback from the mean concentration cells is sparse: the connection probability to granule cells is 0.2 , but if there is a connection, it goes to all three main granule cells. A quantitative, but harder to make sense of, description follows.

Granule cell $\rightarrow$ mitral cell:

$$
w_{i k}^{m g}=w_{m g}\left\{\begin{array}{lr}
1 & \left|3 i-k \bmod N_{g}\right| \leq 1 \\
\xi_{i k}^{m g} & 2 \leq\left|3 i-k \bmod N_{g}\right| \leq 4 \\
0 & 5 \leq\left|3 i-k \bmod N_{g}\right| .
\end{array}\right.
$$

where

$$
\xi_{i k}^{m g}= \begin{cases}1 & \text { probability } p_{m g} \\ 0 & \text { probability } 1-p_{m g}\end{cases}
$$




\begin{tabular}{|c|c|c|}
\hline Parameter & Value & Description \\
\hline$K$ & 640 & number of odors, and number of mean concentration cells $\left(\bar{c}_{j}\right)$ \\
\hline$N$ & 160 & $\begin{array}{l}\text { number of olfactory receptor neuron }(\mathrm{ORN}) \text { receptor types } \\
\text { and number of mitral cells }\end{array}$ \\
\hline$N_{g}$ & 480 & number of granule cells \\
\hline$p_{\text {prior }}$ & $3 / K$ & $\begin{array}{l}\text { prior probability that any particular odor will appear } \\
\text { (see Eq. }(2) \text { ) }\end{array}$ \\
\hline$\beta_{\text {prior }}$ & 3 & prior over the concentration for present odors (see Eq. (2)) \\
\hline$\left(\alpha_{0}, \beta_{0}\right)$ & $(1 / 3,27 / K)$ & $\begin{array}{l}\text { parameters of the Gamma distribution used for } \\
\text { variational inference (see Eq. (10)) }\end{array}$ \\
\hline$\Delta t$ & $50 \mathrm{~ms}$ & time window for counting spikes \\
\hline$\nu_{0, i}$ & variable & $\begin{array}{l}\text { background firing rate, drawn from a Gaussian distribution } \\
\text { mean }=10 \mathrm{~Hz} \text {; standard deviation }=1 \mathrm{~Hz}\end{array}$ \\
\hline$\gamma_{i}$ & variable & $\begin{array}{l}\text { variability parameter, drawn from a } \log \text { normal distribution } \\
\text { mean of } \log \gamma=0.5 ; \text { standard deviation of } \log \gamma=0.275\end{array}$ \\
\hline$\tau_{c}$ & $10 \mathrm{~ms}$ & time constant of mean concentration cells \\
\hline$\tau_{m}$ & $10 \mathrm{~ms}$ & time constant of mitral cells \\
\hline$\tau_{g}$ & $5 \mathrm{~ms}$ & time constant of granule cells \\
\hline$w_{m g}$ & $1 / \sqrt{20}$ & mitral $\leftrightarrow$ granule connection strength \\
\hline$p_{m g}$ & $1 / 2$ & granule cell $\leftrightarrow$ mitral cell connection probability \\
\hline$A_{0}$ & 15 & cortex $\rightarrow$ granule cell connection strength \\
\hline$p_{A}$ & 0.2 & cortex $\rightarrow$ granule cell connection probability \\
\hline$d t$ & $0.1 \mathrm{~ms}$ & time step used in simulations \\
\hline
\end{tabular}

Table 1. Parameters used in the simulations. 
Mitral cell $\rightarrow$ granule cell:

$$
w_{k i}^{g m}=w_{i k}^{m g} .
$$

Piriform cortex $\rightarrow$ granule cell:

$$
A_{k j}=A_{0} \xi_{\lfloor(k+1) / 3\rfloor, j}^{A},
$$

where $\lfloor\cdot\rfloor$ indicates the integer part and

$$
\xi_{j^{\prime} j}^{A}= \begin{cases}1 & \text { probability } p_{A} \\ 0 & \text { probability } 1-p_{A} .\end{cases}
$$

In the above, $i$ and $j$ range from 1 to $N$ and $k$ from 1 to $N_{g}$, both inclusive.

In Sec. 7 we need the typical value of $\beta_{j}$, so here we compute an approximation to it's mean. Given the above definitions, it is straightforward to show that

$$
\left\langle\sum_{i=1}^{N} w_{i j}\right\rangle=3 N w_{m g}^{2}\left(1+2 p_{m g}\right) A_{0} p_{A}=144
$$

where the second equality follows from Table 1 . Combining this with the definition of $\beta_{j}$ (Eq. (22b)), and noting that $\beta_{0}=27 / K$, we see that

$$
\left\langle\beta_{j}\right\rangle \approx \frac{1}{640 / 27+144} \approx \frac{1}{168} .
$$

\section{Determining the probability that an odor is present}

Our inference algorithm takes ORN input and returns a set of $\bar{c}_{j}$, one for each odor. The olfactory system must then turn each $\bar{c}_{j}$ into the probability that odor $j$ is present. This probability can be computed from Bayes' theorem,

$$
p\left(1_{j} \mid \bar{c}_{j}\right)=\frac{p\left(\bar{c}_{j} \mid 1_{j}\right) p\left(1_{j}\right)}{p\left(\bar{c}_{j} \mid 1_{j}\right) p\left(1_{j}\right)+p\left(\bar{c}_{j} \mid 0_{j}\right) p\left(0_{j}\right)}
$$

where $1_{j}$ indicates that odor $j$ is present and $0_{j}$ indicates that it is absent. The prior probability that an odor is present, $p\left(1_{j}\right)$ is just $p_{\text {prior }}$, independent of $j$ (see Eq. (2)). Thus, we need only compute $p\left(\bar{c}_{j} \mid 1_{j}\right)$ and $p\left(\bar{c}_{j} \mid 0_{j}\right)$. To do that, we ran many simulations of Eq. (28) with the number of presented odors and their concentrations drawn from the prior, Eq. (2). The $\bar{c}_{j}$ were evaluated in steady state (at $t=300 \mathrm{~ms}$ ), binned in log space (we used log space because the $\bar{c}_{j}$ can be very small), and the probability distributions were estimated from the histograms. Specifically, letting

$$
\begin{aligned}
& \bar{c}_{j}^{(k)}=\text { value of } \bar{c}_{j} \text { on simulation } k \\
& s_{j}^{(k)}=1 \text { if odor } j \text { was present on simulation } k \text { and } 0 \text { otherwise, }
\end{aligned}
$$


an estimate of the conditional probability distributions over $\log \bar{c}$ is found from

$$
\begin{aligned}
p(\log \bar{c} \mid 1) & =\frac{\sum_{j k} s_{j}^{(k)} \mathbb{1}\left[\log \bar{c}-\delta \log \bar{c} / 2<\log \bar{c}_{j}^{(k)} \leq \log \bar{c}+\delta \log \bar{c} / 2\right]}{\sum_{j k} s_{j}^{(k)}} \\
p(\log \bar{c} \mid 0) & =\frac{\sum_{j k}\left(1-s_{j}^{(k)}\right) \mathbb{1}\left[\log \bar{c}-\delta \log \bar{c} / 2<\log \bar{c}_{j}^{(k)} \leq \log \bar{c}+\delta \log \bar{c} / 2\right]}{\sum_{j k}\left(1-s_{j}^{(k)}\right)}
\end{aligned}
$$

where $\mathbb{1}(\cdot)$ is the indicator function: $\mathbb{1}[x]=1$ if $x$ is true and 0 otherwise. The bin size, $\delta \log \bar{c}$, was 0.0182 . These two distributions were computed from 10,000 trials. Because $p(\log \bar{c})=\bar{c} p(\bar{c})$, we can use the conditional distributions over $\log \bar{c}$ to compute $p(1 \mid \bar{c})$, the probability that odor 1 is present given $\bar{c}$,

$$
p(1 \mid \bar{c})=\frac{\left.p_{\text {prior }} p(\log \bar{c} \mid 1) / p(\log \bar{c} \mid 0)\right)}{\left.1-p_{\text {prior }}+p_{\text {prior }} p(\log \bar{c} \mid 1) / p(\log \bar{c} \mid 0)\right)} .
$$

We used this expression to compute the points in Fig. 3A of the main text. We then fit those points to a sigmoidal function of the form

$$
p(1 \mid \bar{c})=\frac{e^{\kappa\left(\log \bar{c}-\log \bar{c}_{0}\right)}}{1+e^{\kappa\left(\log \bar{c}-\log \bar{c}_{0}\right)}}
$$

where $\kappa=8.3$ and $\bar{c}_{0}=0.31$, chosen to minimize the mean square error between the data and the curve. That's the solid green line in Fig. 3A of the main text.

We used the same data to determine the degree to which the model exhibits concentration invariance: we binned the true concentration, and in each bin computed the mean and standard deviation of the inferred mean concentration, $\bar{c}_{j}$. The results are plotted in Figs. 3B and $\mathrm{C}$ of of the main text. The bins were 0.5 and 0.1 in Figs. $3 \mathrm{~B}$ and $\mathrm{C}$, respectively.

\section{ROC curves}

We constructed three sets of ROC curves, from three different models: one from our simulations (Fig. 8A of the main text), one from a model by Luo et al. ${ }^{12}$ (Fig. 8B of the main text), and one from template matching (Fig. $8 \mathrm{C}$ of the main text). In all three cases, the ROC curves were constructed as follows. The ORN input was mapped to a set of variables, which for now we'll call $z_{j}, j=1, \ldots, K$ (see below for explicit examples). Odor $j$ was then declared to be present if $z_{j}$ was above a threshold and absent if it was below the threshold. For each value of the threshold, we computed the fraction of odors that were declared to be present and actually were present (fraction of true positives), and the number of odors that were declared to be present but were not (number of false positives). These two quantities - fraction of true positives and number of false positive - are plotted on the $y$ and $x$ axes, respectively, in Fig. 8 of the main text.

What differed among the three models was the mapping from ORN activity to $z_{j}$. Our model was simplest: $z_{j}=\bar{c}_{j}$ evaluated at the end of the trial $(300 \mathrm{~ms})$. For Luo at al., ${ }^{12}$ the 
ORN activity on any particular trial was first passed through a nonlinearity to create a new variable, $\rho_{i}$,

$$
\rho_{i}=\frac{r_{i}^{n}}{r_{i}^{n}+\sigma^{n}+\left((m / K) \sum_{i^{\prime}} r_{i^{\prime}}\right)^{n}},
$$

where $n=1.5, \sigma=2$ and $m=0.3$ (chosen to maximize performance). Then, defining

$$
\boldsymbol{\rho} \equiv\left(\rho_{1}, \rho_{2}, \ldots, \rho_{N}\right)
$$

(recall that $N$ is the number of ORN receptor types; see Table 1 ), $z_{j}$ was given, on any particular trial, by

$$
z_{j}=\frac{\left(\boldsymbol{\rho}^{j}-\overline{\boldsymbol{\rho}}\right) \cdot \mathbf{C}^{-1} \cdot \boldsymbol{\rho}}{\left(\boldsymbol{\rho}^{j}-\overline{\boldsymbol{\rho}}\right) \cdot \mathbf{C}^{-1} \cdot \boldsymbol{\rho}^{j}}
$$

where "." indicates the standard dot product, $\boldsymbol{\rho}^{j}$ is the mean activity associated with odor $j$,

$$
\rho_{i}^{j}=\int d c_{j} \frac{e^{-c_{j} / \beta_{\text {prior }}}}{\beta_{\text {prior }}} \frac{\left(w_{i j} c_{j}\right)^{n}}{\left(w_{i j} c_{j}\right)^{n}+\sigma^{n}+\left((m / K) \sum_{i^{\prime}} w_{i^{\prime} j} c_{j}\right)^{n}},
$$

and $\overline{\boldsymbol{\rho}}$ and $\mathbf{C}$ are the mean and covariance of $\boldsymbol{\rho}$,

$$
\begin{aligned}
\overline{\boldsymbol{\rho}} & =\frac{1}{K} \sum_{j=1}^{K} \boldsymbol{\rho}^{j} \\
\mathbf{C} & =\frac{1}{K} \sum_{j=1}^{K}\left(\boldsymbol{\rho}^{j}-\overline{\boldsymbol{\rho}}\right)\left(\boldsymbol{\rho}^{j}-\overline{\boldsymbol{\rho}}\right) .
\end{aligned}
$$

For template matching, $z_{j}$ was taken to be the cosine of the angle between the vector of ORN responses, $\mathbf{r} \equiv\left(r_{1}, r_{2}, \ldots, r_{N}\right)$, and the vector of weights $\mathbf{w}_{j} \equiv\left(w_{1 j}, w_{2 j}, \ldots, w_{N j}\right)$,

$$
z_{j}=\frac{\mathbf{r} \cdot \mathbf{w}_{j}}{\left[(\mathbf{r} \cdot \mathbf{r})\left(\mathbf{w}_{j} \cdot \mathbf{w}_{j}\right)\right]^{1 / 2}} .
$$

For activity, $\mathbf{r}$, we used spike count evaluated $50 \mathrm{~ms}$ after odor onset.

\section{$7 \quad$ Regularized models}

Our model can be written

$$
\overline{\mathbf{c}}=\underset{\overline{\mathbf{c}}^{\prime}}{\operatorname{argmax}}\left[\mathcal{L}\left(\overline{\mathbf{c}}^{\prime}\right)+\Omega\left(\overline{\mathbf{c}}^{\prime}\right)\right]
$$

where

$$
\begin{aligned}
& \mathcal{L}(\overline{\mathbf{c}})=\sum_{i}\left[r_{i} \log \left(\Delta t \nu_{0, i}+\sum_{j} w_{i j} F_{j}\left(\bar{c}_{j}\right)\right)-\sum_{j} w_{i j} F_{j}\left(\bar{c}_{j}\right)\right] \\
& \Omega(\overline{\mathbf{c}})=-\sum_{j}\left[\psi\left(\bar{c}_{j} / \beta_{j}\right)\left(\bar{c}_{j} / \beta_{j}-\alpha_{0}\right)-\log \Gamma\left(\bar{c}_{j} / \beta_{j}\right)-\sum_{i} w_{i j} F_{j}\left(\bar{c}_{j}\right)\right] .
\end{aligned}
$$


To show this, differentiate $\mathcal{L}(\overline{\mathbf{c}})+\Omega(\overline{\mathbf{c}})$ with respect to $\bar{c}_{j}$ and set the resulting expression to zero; that yields Eq. (25).

The first term, $\mathcal{L}(\overline{\mathbf{c}})$, is the $\log$ likelihood (the $\log$ of Eq. (6)), but with $c_{j}$ replaced by $F_{j}\left(\bar{c}_{j}\right)$ (Eq. (26)), which is the geometric mean under the Gamma distribution (Eq. (21a)),

$$
F_{j}\left(\bar{c}_{j}\right)=e^{\left\langle\log c_{j}\right\rangle} .
$$

The second term, $\Omega(\overline{\mathbf{c}})$, is a regularizer, in the sense that when $\alpha_{0}$ is small, it pushes $\bar{c}_{j} / \beta_{j}$ toward $\alpha_{0}$. To understand how it behaves in that limit, note that when $\bar{c}_{j} / \beta_{j} \rightarrow 0$,

$$
\begin{aligned}
\Gamma\left(\bar{c} / \beta_{j}\right) & \rightarrow \frac{\beta_{j}}{\bar{c}_{j}} \\
\psi\left(\bar{c} / \beta_{j}\right) & \rightarrow-\frac{\beta_{j}}{\bar{c}_{j}} .
\end{aligned}
$$

The second expression, combined with the definition of $F_{j}\left(\bar{c}_{j}\right)$ given in Eq. (26), implies that $F_{j}\left(\bar{c}_{j}\right) \rightarrow 0$ as $\bar{c}_{j} / \beta_{j} \rightarrow 0$. Consequently, when both $\alpha_{0}$ and $\bar{c}_{j} / \beta_{j}$ are small,

$$
\Omega(\boldsymbol{\alpha}) \rightarrow \sum_{j}\left[\frac{\bar{c}_{j} / \beta_{j}-\alpha_{0}}{\bar{c}_{j} / \beta_{j}}-\log \left(\bar{c}_{j} / \beta_{j}\right)\right] .
$$

Each term in brackets has a quadratic peak, with the $j^{\text {th }}$ term peaking at $\bar{c}_{j}=\beta_{j}$. The fact that the peaks are quadratic makes this different from an L1 regularizer, which would typically drive $\bar{c}_{j}$ all the way to 0 . Nevertheless, this regularizer pushes $\bar{c}_{j}$ close to (but slightly above) $\beta_{j} \alpha_{0}$, which is close to zero (it's about 0.002 ). The peaks have a width of $\beta_{j} \alpha_{0}$; given that $\alpha_{0}=1 / 3$ (see Table1) and $\beta_{j}$ is about $1 / 168$ (see Eq. (38)), the peaks are relatively narrow, and can correspond to sharp local maxima. We chose $\alpha_{0}=1 / 3$ to avoid avoid getting stuck in them.

Both Koulakov and Rinberg ${ }^{13}$ and Tootoonian and Lengyel ${ }^{4}$ also cast olfaction as a minimization problem. For both of their models, the "likelihood" was quadratic,

$$
\mathcal{L}(\mathbf{x})=\frac{1}{2 \epsilon} \sum_{i}\left(r_{i}-\sum_{j} w_{i j} x_{j}\right)^{2},
$$

and the regularizer had the form

$$
\Omega(\mathbf{x})=\sum_{j} \theta_{j} x_{j} \Theta\left(x_{j}\right) .
$$

The variables, $x_{j}$, had different interpretations in the two models: for Koulakov and Rinberg it was granule cell activity, and for Tootoonian and Lengyel it was a binary variable signaling the presence or absence of chemicals. In addition, for Tootoonian and Lengyel's model, $\epsilon \rightarrow 0$ (to enforce the constraint exactly), whereas for Koulakov and Rinberg's model, $\epsilon$ was finite.

Druckmann et al. considered a very similar model, except they applied it to arbitrary stimuli rather than specifically to olfaction. ${ }^{14}$ Their model used the same likelihood as in 
Eq. (55), with, like Tootoonian and Lengyel, $\epsilon \rightarrow 0$. The regularizer was a combination of L1 and L2 norms,

$$
\Omega(\mathbf{x})=\sum_{j}\left|x_{j}\right|+\frac{1}{2 \delta} \sum_{i} x_{i}^{2} .
$$

Unlike in the work targeted specifically for olfaction, ${ }^{4,13}$ in Druckmann et al.'s model the $x_{j}$ were not constrained to be positive.

\section{Code availablity}

All experimental procedures were based on a custom software, written in Matlab (R2013a) environment. The code is available as a zipped archive at

http://www.gatsby.ucl.ac.uk/ pel/olfaction.zip

The readme file contains instruction on how to run the code.

The code uses a different random number seed for each run, so the results will differ slightly from the figures in the paper. This could have been avoided by fixing the random number generator. However, we preferred not to risk drawing conclusions that would not generalize over random seeds. The exact parameters used to generate our figures (connectivity matrix, scaling factors and baseline firing rates) can be obtained on request (email agnieszka.grabskabarwinska@gmail.com).

\section{References}

[1] Erdi, P., Gröbler, T., Barna, G. \& Kaski, K. Dynamics of the olfactory bulb: bifurcations, learning, and memory. Biological Cybernetics 69, 57-66 (1993).

[2] Hendin, O., Horn, D. \& Tsodyks, M. V. Associative memory and segmentation in an oscillatory neural model of the olfactory bulb. J. Comput. Neurosci. 5, 157-169 (1998).

[3] Rokni, D., Hemmelder, V., Kapoor, V. \& Murthy, V. N. An olfactory cocktail party: figure-ground segregation of odorants in rodents. Nature Neurosci. 17, 1225-1232 (2014).

[4] Tootoonian, S. \& Lengyel, M. A dual algorithm for olfactory computation in the locust brain. In Advances in Neural Information Processing Systems 27, 2276-2284 (Curran Associates, Inc., 2014).

[5] Polese, D., Martinelli, E., Marco, S., Di Natale, C. \& Gutierrez-Galvez, A. Understanding odor information segregation in the olfactory bulb by means of mitral and tufted cells. PloS one 9, e109716 (2014).

[6] Firestein, S. \& Werblin, F. Odor-induced membrane currents in vertebrate-olfactory receptor neurons. Science 244, 79-82 (1989). 
[7] Wachowiak, M. \& Cohen, L. B. Representation of odorants by receptor neuron input to the mouse olfactory bulb. Neuron 32, 723-735 (2001).

[8] Bozza, T., Feinstein, P., Zheng, C. \& Mombaerts, P. Odorant receptor expression defines functional units in the mouse olfactory system. J. Neurosci. 22 (2002).

[9] Grosmaitre, X., Vassalli, A., Mombaerts, P., Shepherd, G. M. \& Ma, M. Odorant responses of olfactory sensory neurons expressing the odorant receptor mor23: a patch clamp analysis in gene-targeted mice. Proc. Natl. Acad. Sci. 103, 1970-1975 (2006).

[10] Mitchell, T. \& Beauchamp, J. Bayesian variable selection in linear regression. Journal of the American Statistical Association 83, 1023-1032 (1988).

[11] Wainwright, M. J. \& Jordan, M. I. Graphical Models, Exponential Families, and Variational Inference (Foundations and Trends Machine Learning) (Now Publishers Inc, 2008).

[12] Luo, S. X., Axel, R. \& Abbott, L. F. Generating sparse and selective third-order responses in the olfactory system of the fly. Proc. Natl. Acad. Sci. 107, 10713-10718 (2010).

[13] Koulakov, A. A. \& Rinberg, D. Sparse incomplete representations: a potential role of olfactory granule cells. Neuron 72, 124-136 (2011).

[14] Druckmann, S., Hu, T. \& Chklovskii, D. B. A mechanistic model of early sensory processing based on subtracting sparse representations. In Advances in Neural Information Processing Systems 25, 1979-1987 (Curran Associates, Inc., 2012). 